\title{
Handbuch Landesgeschichte
}

Herausgegeben von

Werner Freitag, Michael Kißener, Christine Reinle, Sabine Ullmann

\section{DE GRUYTER}

OLDENBOURG 


\section{Migration und Minderheiten in Mittelalter und Neuzeit}

\section{Einleitung}

Migrationsgeschichte, in Deutschland vornehmlich im Sinne Klaus J. Bades als „sozialhistorische Migrationsforschung" verstanden, ${ }^{1}$ ist zunächst nicht als Thema der landesgeschichtlichen Forschung konzipiert; im Mittelpunkt steht vielmehr das Anliegen, großräumige, oft sogar globale Zusammenhänge zu erfassen. Themen der Migrationsgeschichte lassen sich dennoch vielfach landeshistorisch fruchtbar machen.

Migration stellt als Bewegung von Menschen im Raum in der Regel einen lebenslangen, oft sogar generationenübergreifenden Prozess dar und ist, anders als Mobilität, meist mit dem Überschreiten von politischen oder kulturellen Grenzen verbunden sowie mit der Absicht eines längeren, wenn auch nicht zwingend permanenten Verbleibs am Zielort. ${ }^{2}$ Die Gründe waren schon immer vielfältig: Hunger, Krieg, politische und religiöse Verfolgung konnten wie auch Kriegszüge mit Landnahme, Kolonisierung, Arbeitssuche oder die Suche nach besseren Lebensbedingungen Migrationen auslösen. Migranten brachten ihre Kenntnisse und Fähigkeiten in die neue Heimat mit und trugen zu Kulturtransfer und Verflechtung bei. Zuwanderung wurde oft zum Motor für die Entwicklung der Städte und des Landes; vielfach wurde sie auch mit der Erwartung einer solchen Entwicklung verbunden und daher obrigkeitlich gefördert. Migration und ihre Rahmenbedingungen waren. somit immer auch geprägt von den jeweils vorherrschenden Migrationsregimen, also der Gesamtheit der Einstellungen, Haltungen und Regulierungsmechanismen in Bezug auf Migration: ${ }^{3}$ Diese waren restriktiv oder liberal, sie hemmten oder förderten Migration, ohne dass sie freilich je ganz von obrigkeitlichen Normsetzungen kontrolliert werden konnten.

Migranten sind nicht automatisch Angehörige von Minderheiten, doch sie werden oft marginalisiert und bekommen einen Minderheitenstatus zugeschrieben. Umgekehrt waren Minderheiten - oft religiöse Minderheiten wie die Juden oder ethnische bzw. als ethnisch empfundene Minderheiten wie die Sinti und Roma - immer wieder auf Migration als Überlebensnische angewiesen oder wurden durch Vertreibungen zu Migranten. In der Frühen Neuzeit spielte zudem die staatliche Förderung von Migration aufgrund utilitaristischer Motive eine wichtige Rolle. Durch entspre-

\footnotetext{
1 Bade, Migrationsforschung 2004.

2 Gestrich/Krauss, Migration 1998.

3 Oltmer, Einführung 2009.
} 
chende Privilegien konnten Migranten hier sogar als wirtschaftliche, geistig und politische Eliten fungieren.

Im Rahmen von Traditionsbildungsprozessen in den Ländern und Regionen haben Migranten - mit wenigen Ausnahmen wie etwa den Hugenotten - allerdings oft noch keinen Platz. Bis heute gelten sie eher als Gefahr für die bestehende Ordnung, stehen sie doch im Gegensatz zu einer Norm, für die Sesshaftigkeit den Normalfall, Bewegung im Raum indes eine Irregularität darstellt. Insbesondere in den Städten wurden und werden Migranten vielfach als Problemgruppe wahrgenommen und beschrieben, obwohl Städte Zeit ihres Bestehens von und mit ihnen lebten.

Das Interesse an Migrationen lag im 19. Jahrhundert bei der Nationalökonomie, die später mit der „Bevölkerungsforschung“ eine enge Verbindung einging. Ziel war die Kenntnis über aktuelle Wanderungsbewegungen, die auch vor dem Hintergrund von Bevölkerung und Arbeitsmarkt als politisch und gesellschaftlich relevant galten. $\mathrm{Ab}$ dem 20. Jahrhundert interessierten sich Soziologie und Geschichtswissenschaft für Migrationsthemen, in den letzten Jahrzehnten ergänzt durch Ethnologie, Sozialpsychologie, Pädagogik, Literaturwissenschaft und Religionswissenschaft. Historische Migrationsforschung ist demnach heute prinzipiell interdisziplinär ausgerichtet. Hier lässt sich ein Bogen schlagen zur Landesgeschichte, die ja ebenfalls genuin interdisziplinär angelegt ist und aus der Methodenvielfalt ihren besonderen Zugriff auf lokale und regionale Entwicklungen ableitet.

Durch einen lokal-regional fokussierten Blickwinkel kann die Landesgeschichte Bewegungen und ihre Auswirkungen ,vor Ort' untersuchen. Der Fokus liegt dann eher auf den Migrationsregimen, die den Wandel mitbestimmten, sowie auf den konkreten Auswirkungen in der Stadt oder im ländlichen Raum. Gegenstand landesgeschichtlicher Untersuchungen sind weiterhin die Lebensbedingungen von zugewanderten Minderheiten in lokalen und regionalen Zusammenhängen sowie die Haltung der jeweiligen Mehrheitsgesellschaft zu diesen Gruppen. Gerade hier - im Blick auf die lokale Situation - bietet sich auch die Chance der differenzierenden Betrachtung, die traditionelle Narrative hinterfragt.

Die folgenden Ausführungen können hier nur exemplarisch vorgehen. Anhand der mehr oder weniger den heutigen Bundesländergrenzen entsprechenden Großräume Bayern und Brandenburg werden Migrationen und Minderheiten thematisiert, mit unterschiedlichen Zugriffen und unterschiedlichen Schwerpunktsetzungen. Aus einer vergleichenden Perspektive lässt sich konstatieren, dass das Migrationsgeschehen in beiden Großregionen zumindest bis ins 20. Jahrhundert hinein sehr stark von ähnlichen Faktoren geprägt wurde, die sich aber sowohl regional als auch zeitlich unterschiedlich auswirkten. Erschwert wird ein solcher Vergleich über einen längeren Zeitraum natürlich durch die Tatsache, dass diese Regionen historisch territorial vielfältig waren und über die Jahrhunderte wechselnde Zugehörigkeiten aufwiesen.

An den großen, konfessionell induzierten Migrationsbewegungen hatte Brandenburg sicher mit Blick auf eine gezielte Aufnahmepolitik einen weitaus größeren 
Anteil als Bayern, während die hohenzollernschen Territorien in Franken, in denen die Aufnahme vergleichbar war, damals eben nicht zu Bayern gehörten. In Franken lassen sich auch erinnerungskulturell - etwa in Bezug auf die Hugenottenansiedlung - durchaus Ähnlichkeiten mit Brandenburg feststellen, die im historischen Bayern in dieser Form fehlen. Im Zuge der frühmodernen Staatsbildungsprozesse kam es in Bayern wie in Brandenburg zur Ausweisung von Minderheiten. Der Umgang etwa mit Angehörigen der jüdischen Religion zeigt im regionalen Vergleich aber die Variationsbreite der obrigkeitlichen Verhaltensweisen: Während Kurbayern, mit Ausnahme einer kleinen Hofjudengemeinde in München, am restriktiven Kurs festhielt, verfolgte Kurbrandenburg nach der Mitte des 17. Jahrhunderts in ähnlicher Weise wie die hohenzollernsche Linie in Franken eine Judenschutzpolitik.

Für das 20. Jahrhundert und hier besonders für die Zeit nach dem Zweiten Weltkrieg zeigt sich bei der Aufnahme von Heimatvertriebenen ein deutlicher Systemunterschied: Die erzwungene Aufnahme und Eingliederung der Zwangsmigranten aus den ehemals deutschen Ostgebieten und dem östlichen Europa spielte zweifellos in beiden Regionen eine enorme Rolle; doch während im Westen und damit auch in Bayern die Vertriebenen ihre eigene Kultur weiterhin pflegen und sich seit der Gründung der Bundesrepublik auch landsmannschaftlich organisieren konnten, war dies in der SBZ/DDR nicht der Fall. Unter der offiziellen Decke der Assimilation fand dort jedoch eine erinnerungskulturelle Weitergabe statt, die sich bereits vor dem Mauerfall in einer umfänglichen literarischen Verarbeitung niederschlug und sich nach der Wiedervereinigung wieder deutlich Raum schuf. Mindestens ebenso stark wie die Systemunterschiede prägten jedoch, wie Studien für Sachsen und Mecklenburg-Vorpommern wie für Niedersachsen und Hamburg zeigen, StadtLand-Unterschiede den Integrationsprozess. Im Systemvergleich werden erneut die Paradigmen "Arbeit“, „Chancen“ und „Kultur" als wichtige Elemente auf dem Weg des Ankommens und Sesshaft-Werdens erkennbar.

\section{Bayern, Franken und Schwaben}

\section{Marita Krauss}

Migration war bisher eher ein Stiefkind der Bayerischen Landesgeschichte. Sesshaftigkeit und Stabilität politischer wie gesellschaftlicher Strukturen werden meist selbstverständlich als das „Normale“ angenommen, Migration und ihre Folgen als der Sonderfall. Die Frage nach Migrationen richtet sich jedoch auf gesellschaftlichen Wandel und tritt an die Sesshaften gewissermaßen von der Seite heran; sozial- und kulturgeschichtliche Migrationsgeschichte untersucht eine Gesellschaft auch und gerade in ihrem Umgang mit dem Fremden, dem sozial nicht Konformen. Das gilt auch für die Geschichte von Minderheiten, die wie „die Fremden“ oft Ablehnung und Hass der Ansässigen auf sich zogen. Auswanderung, Zuwanderung und 
Binnenmigration brachten zu allen Zeiten die Gesellschaft in Bewegung und veränderten die Wahrnehmung der umgebenden Welt. Migration ist daher gerade für die Landesgeschichte ein wichtiges Untersuchungsfeld, lassen sich doch etliche dieser Themen nur am lokalen und regionalen Beispiel fundiert bearbeiten.

Im Folgenden sollen einige wichtige Linien des Themas am Beispiel Bayerns gezeigt werden. Das heutige Bundesland Bayern, dessen Grenzen hier maßgeblich sein werden, umfasst neben den altbayerischen Territorien die Teile der historischen Regionen Schwaben und Franken, die zu Beginn des 19. Jahrhunderts an das Königreich Bayern fielen. Die bayerische Rheinpfalz, ein wichtiges Emigrationsland, nahm wegen der besonderen politischen und geographischen Lage vielfach eine ganz eigene Entwicklung und soll daher hier nicht berücksichtigt werden.

\subsection{Das ius emigrandi und die Folgen}

Bereits die Eroberung, Besiedelung und Erschließung des Landes im Mittelalter ist nicht ohne Migration denkbar. Das spätere Bayern war immer wieder Schmelztiegel und Durchzugsgebiet verschiedenster Völker. ${ }^{4}$ Migrationen prägten in unterschiedlichem Maße Kriegszüge und Eroberungen, Missionsaktivitäten und Klostergründungen, Rodungen und Landesausbau. Städte waren bis ins 18. Jahrhundert in der Regel auf demographische Ergänzungen angewiesen, die Entwicklung von Handwerk, Kunst und Wissenschaft wurde stark von Migrationen geprägt und vorangetrieben. Mit der zunehmenden Territorialisierung von Herrschaft seit dem Spätmittelalter ging vielfach der Versuch einher, Mobilität zu kontrollieren und sie zumindest für produktive und erst recht für hoch qualifizierte Personengruppen (etwa Studenten und Gesellen) einzuschränken. Auf der anderen Seite ist auch die zunehmende Kriminalisierung von Vaganten im Rahmen der "guten Policey“ in diesem Zusammenhang zu sehen. Die Entwicklung verfestigter territorialer Grenzen gehört fraglos zu den Fundamentalvorgängen der Frühneuzeit.

Reformation und Konfessionalisierung akzentuierten die Bedeutung der territorialen Grenzen und bedingten andererseits neue Formen der Migration. Sie hatten zur Folge, dass nicht wenige territoriale Grenzen als Konfessionsgrenzen mit neuer Bedeutung aufgeladen wurden; sie begünstigten die Herausbildung "staatlicher“ Steuerungsinstrumente, die im Sinne einer konfessionellen Homogenisierung des Herrschaftsverbandes genutzt wurden - und sie hatten zur Folge, dass sich mit der Konfessionsmigration ein neuer Migrationstypus entwickelte. Diese Ambivalenz wird nicht zuletzt am ius emigrandi des Augsburger Religionsfriedens von 1555 deutlich. Dies schrieb zwar eine Schutzklausel vor religiös bestimmter Verfolgung fest, sie korrespondierte jedoch mit dem landesherrlichen Recht, die Religion der Unter-

4 Prinz, Geschichte 1997. 
tanen zu bestimmen und anderskonfessionelle Untertanen zur Auswanderung zu zwingen. ${ }^{5}$

Auf das Recht zur Auswanderung beriefen sich die evangelischen Glaubensflüchtlinge, die seit den 1590er Jahren nach Entrichtung einer zehnprozentigen Nachsteuer auf ihren Besitz aus den österreichischen Erblanden flohen, um sich vor allem im süddeutschen Raum wieder anzusiedeln. Der Einwanderungsprozess vollzog sich in mehreren Wellen und die Aufnahmebedingungen blieben keineswegs konstant. ${ }^{6}$ Entscheidend für die Integration dieser Glaubensflüchtlinge waren deren ökonomische Verhältnisse: Wirtschaftlich potente Zuwanderer mit guten geschäftlichen Verbindungen fanden, vor allem in Friedenszeiten, deutlich schneller Aufnahme als arme Migranten, die bei den Ansässigen die Sorge auslösten, sie könnten der finanziellen Unterstützung bedürfen. Die rechtliche Integration dieser wohlhabenden Immigranten wurde meist in kurzer Zeit vollzogen. Die gemeinsame Konfession und die gemeinsame Sprache waren dafür von großer Bedeutung, senkten sie doch auch die Bedenken gegenüber einer möglichen Einheirat, dem Konnubium, das bis heute als zentrales Integrationskriterium gilt. Von Bedeutung war überdies das bewegende Schicksal der Glaubensflüchtlinge, das bei den Ansässigen eine emotionale Integrationsbereitschaft schuf. ${ }^{7}$ Auf positive Aufnahme konnten Flüchtlinge auch rechnen, wenn sie als Arbeitskräfte gebraucht wurden. So ging es den Immigranten der 1650er Jahre, als in den ländlichen Gemeinden Westmittelfrankens deutlich über 100.000 Menschen Aufnahme fanden; sie machten mehr als ein Drittel der Bevölkerung aus. ${ }^{8}$ Die Kriegsverluste des Dreißigjährigen Krieges waren sehr hoch gewesen und die nun einwandernde bäuerliche Bevölkerung wurde dringend gebraucht, um die oft seit Jahren nicht mehr kultivierten Höfe wieder zu bewirtschaften. Die Einwanderer nach Franken kamen dabei oftmals aus den Böhmischen Ländern; Schwaben wurde von Tirol aus neu besiedelt. Die Grund- und Territorialherren rissen sich um die Neuankömmlinge und stellten ihnen zu günstigsten Bedingungen Land zur Verfügung. Krieg und Not senkten jedoch deutlich die Bereitschaft, Fremde aufzunehmen. Als während des Dreißigjährigen Krieges Flüchtlinge aus dem mittleren und einfachen Bürgertum hofften, in Reichsstädten wie Nürnberg aufgenommen $\mathrm{zu}$ werden, stießen sie auf Ablehnung. Die Städte erhöhten die finanziellen Hürden des Zuzugs drastisch. Die Aufnahmegesellschaft, so ist das zu resümieren, bestimmte die Bedingungen. Soziale und wirtschaftliche Integration der Immigranten hing maßgeblich von diesen Vorgaben ab.

Doch die Aufnahmegesellschaft profitierte, entgegen anfänglicher Befürchtungen, am meisten von Zuwanderung. Dies zeigt sich auch am Beispiel der zentralen

5 Gotthard, Religionsfrieden 2004, S. 119.

6 Schnabel, Exulanten 1992.

7 Schnabel, Glaubensflüchtlinge 2003, S. 163.

8 Ebd., S. 168. 
Glaubensmigration der Folgezeit. ${ }^{9}$ Die Zuwanderung von Hugenotten nach der Aufhebung des Edikts von Nantes durch Ludwig XIV. im Jahr 1685 legte durch die Gründung von „Christian-Erlang“ den Grundstein für die wirtschaftliche Prosperität des Ortes Erlangen. Bald folgten auch Kurpfälzer Exulanten, die vor den Raubzügen Ludwigs XIV. geflohen waren. Über viele Jahre war Erlangen zweisprachig.

Auch Arbeitsmigration mit temporärer Ortsverlagerung gab es bereits im 17. Jahrhundert. Dazu gehörten die „Schwabenkinder“, deren jährliche Züge in den oberschwäbischen Bereich seit 1625 nachgewiesen sind. So berichtete Johann Conrad Kostner, Verwalter auf Schloss Bludenz, an die Regierung in Innsbruck: „wol ziehen alle Jahr zu Frühlingszeiten viel Kinder auf die Hüt nacher Ravensburg, Überlingen und ins Reich hin und wieder, welche aber vor und nach Martini zu Hause kommen." ${ }^{10}$ Höhepunkte dieser Wanderbewegungen lagen im 19. Jahrhundert: Schätzungen gehen von 4.000 Kindern jährlich aus, die als Hütekinder nach Oberschwaben zogen und sich dort den Sommer über verdingten, um ihre armen Eltern in Graubünden, im Montafon, in Tirol zu entlasten. Die 5- bis 14-jährigen Schwabenkinder kamen aus Realteilungsgebieten; sie stammten aus kinderreichen Familien, die an der Armutsgrenze lebten. Auch die katholischen Gegenden des Allgäus waren Wanderziele der Schwabenkinder, die selbst aus einem katholischen Umfeld stammten.

\subsection{Die jüdische Minderheit}

Minderheiten, die der Verfolgung ausgesetzt waren und daher mehrfach den Ort wechseln mussten, gehörten zwangsweise meist zu den mobilen Bevölkerungsgruppen. Die bedeutendste dieser Minderheiten waren die Juden. In Altbayern entstanden zunächst in Regensburg, im 13. Jahrhundert auch in Städten wie München erste jüdische Gemeinden, deren Mitglieder im Fernhandel und im Geldverleih tätig waren. Nach Pogromen, erneuter Ansiedlung und Vertreibung erließen die bayerischen Herzöge strenge Aufenthalts- und Durchreiseverbote für Juden. Erst in der zweiten Hälfte des 17. Jahrhunderts gab es wieder eine jüdische Gemeinde in Regensburg. Als Bankiers der Kurfürsten und des ersten bayerischen Königs erhielten Hoffaktorenfamilien im 18. und noch im beginnenden 19. Jahrhundert eine wichtige Stellung. ${ }^{11}$ Dazu gehörte die Familie Seligmann; Aaron Elias Seligmann wurde als erster Jude von König Max I. Joseph als Freiherr von Eichthal in den Adelsstand erhoben. Er konvertierte erst auf dem Totenbett. Die international tätige Familie Eichthal spielte im 19. Jahrhundert noch eine zentrale Rolle bei der Gründung der Baye-

9 Kluxen/Krieger/May, Fremde 2016; Friederich, Hugenottenstadt 1986.

10 Uhlig, Schwabenkinder ${ }^{4} 2003$.

11 Treml/Kirmeier, Geschichte 1988. 
rischen Hypotheken- und Wechselbank, der wichtigsten Bank zur Finanzierung des Agrarkredits in Bayern.

Auch im heutigen bayerischen Schwaben entstanden im 13. Jahrhundert die ersten jüdischen Gemeinden in den Städten, vor allem in der Reichsstadt Augsburg. ${ }^{12}$ Ihre Lebensumstände wurden weitgehend vom städtischen Rat bestimmt, obwohl sie formal Kaiser und Reich unterstanden. Wie anderswo ernährten sie sich überwiegend durch Warenhandel und Geldverleih. Die Verfolgungen im Zuge der „Rintfleischpogrome“ des späten 13. Jahrhunderts und der großen Pest von 1348 bis 1351 bedeuteten einen tiefen Einschnitt. Obwohl es unter Karl IV. zu einer schnellen Wiederansiedlung kam, verschlechterte sich ihre rechtliche und ökonomische Situation. Vorwürfe von Brunnenvergiftung und Hostienschändung sowie Ritualmordverdächtigungen gehörten $z u$ den antijüdischen Stereotypen der Zeit. Immer wieder kam es zu Vertreibungen. Im Laufe des 15. und 16. Jahrhunderts entstanden als Folge mehrerer Migrationsschübe jüdische Gemeinden im ländlichen Gebiet rund um Augsburg sowie im 17. Jahrhundert in großer Verdichtung in der Grafschaft Oettingen und der Markgrafschaft Burgau. Während des 18. Jahrhunderts stieg der jüdische Anteil in einigen dieser Dörfer auf 30 bis 40\%. Obwohl das "Judenedikt" von 1813 die weitgehende rechtliche Gleichstellung der Juden in Bayern brachte, wurde erst 1871 mit dem Eintritt in das Deutsche Reich die vollständige Emanzipation erreicht.

\subsection{Die große Auswanderung}

Im 18. und 19. Jahrhundert wurde die Auswanderung zu einem wichtigen Thema. Greifbar ist vor allem die Entwicklung im Kurfürstentum und im späteren Königreich Bayern, da sich die Vorgänge in den zahlreichen kleinen und kleinsten Territorien Frankens und Schwabens vor der Zugehörigkeit zu Bayern schwer fassen lassen. In Bayern wurde in einem langsamen Prozess das Abwandern der Landeskinder akzeptiert, das - von wenigen staatlich abgesegneten Auswanderungsunternehmungen abgesehen - bis ins 19. Jahrhundert unter Strafe stand. Entlang dieser Haltung zur Mobilität lässt sich im ersten Drittel des 19. Jahrhunderts eine Schnittstelle zwischen der alten und der neuen Zeit erkennen: Zunächst galten die Untertanen noch als Besitz des Grundherrn und in erweitertem Sinne des Landesherrn, als Abhängige, die ohne Erlaubnis und Abstandszahlung ihren Ort nicht verlassen durften. Die Mobilisierung der Gesellschaft in der Folge der Französischen Revolution, der Napoleonischen Kriege und der Montgelas'schen Reformen begann diese Auffassung langsam aufzuweichen. ${ }^{13}$

12 Ullmann, Nachbarschaft 1999; Fassl, Geschichte 1994-2007.

13 Raithel, Auswanderung 2004. 
Auswanderung, legale und illegale, hatte es jedoch in Bayern bereits vorher häufig gegeben: ${ }^{14}$ Die erste Verordnung dazu stammt aus dem Jahr 1644, als es galt, die konfessionell motivierte Auswanderung nach dem Dreißigjährigen Krieg zu steuern. Kolonisten wurden für Russland angeworben und per Schiff zog es Ende des 18. Jahrhunderts viele Bayern über die Donau nach Ungarn - eine der wenigen Auswanderungen, die von den bayerischen Behörden geduldet wurde. Südosteuropa zog viele Siedler an, es bestand jedoch auch bereits über den Rhein, über Antwerpen und Rotterdam eine etablierte Auswanderungsroute nach Amerika. Doch Untertanen waren nach den großen Verlusten des Dreißigjährigen Krieges in erster Linie eine fiskalische Ressource. Noch wichtiger war ihr Geld und Gut, das sie durch eine Auswanderung dem Heimatland zu entziehen drohten, weshalb Auswanderungswillige auch mit einer beträchtlichen Steuer, einem „Abzugsgeld“, belegt wurden. Wer ohne Erlaubnis des Landesherrn auswanderte, musste mit schärfsten Strafen rechnen, wenn er gefasst wurde oder später wieder einreiste: Es drohten Vermögenskonfiszierung und Arbeitshaus. Gegen Ende des 18. Jahrhunderts gab es zunächst für Andersgläubige, Juden und Bettler eine Lockerung des Verbots. In der bayerischen Verfassung von 1818 wurde dann ein beschränkter Auswanderungsanspruch zumindest in andere deutsche Staaten festgeschrieben. ${ }^{15}$ Erst ab 1871 gab es ein Recht auf Auswanderung. ${ }^{16}$

Die Gründe, das Land zu verlassen, waren vielfältig und blieben bis weit in die zweite Hälfte des 19. Jahrhunderts hinein ähnlich. Anfangs dominierte die religiöse Motivation, diese nahm aber immer mehr ab. Doch gerade für Mitglieder protestantischer Sekten blieb die Auswanderung attraktiv, ebenso für Juden vor allem aus Schwaben, der Pfalz und Franken: Immerhin wird die Zahl der jüdischen Auswanderer aus dem bayerischen Gebiet bis 1871 auf 20.000 bis 25.000 geschätzt. ${ }^{17}$ Aber auch Hunger oder kriegerische Ereignisse, hohe Steuern, wachsender Bevölkerungsdruck, eine sich grundlegend wandelnde Arbeitswelt und die zunehmende Mobilität infolge der Bauernbefreiung gehörten zu den Motiven und damit die Suche nach Arbeit und besserem Verdienst, nach einer Umgehung des Wehrdienstes, aber auch nach der Chance, zu heiraten und auf eigenem Grund und Boden sesshaft zu werden - was in Bayern bis 1868 für wenig Bemittelte strengsten Vorschriften unterlag. Hinzu kam aber vor allem die Attraktivität der Alternativen im Auswanderungsland. Auch Abenteuerlust spielte eine nicht zu unterschätzende Rolle.

Einen ersten Schub erfuhr die Auswanderung aus dem südlichen Deutschland in den Jahren 1816/17, als auf Missernten eine große Hungersnot folgte. Dies betraf

14 Bade/Oltmer, Deutschland 2007, S. 143.

15 Verfassungsurkunde des Königreichs Bayern 1818, Tit. IV § 14, zit. nach URL: http://www.verfassungen.de/de/by/bayern18-index.htm [15.02.2017].

16 Rottmann, Lehre 1862.

17 Brinkmann, Gemeinde 2002, S. 49-59. 
vor allem Südwestdeutschland; aber auch die Auswanderung aus Armutsgebieten des neuen Bayern stieg an. $\mathrm{Zu}$ weit größeren Auswanderungswellen kam es in der Jahrhundertmitte und noch einmal im letzten Drittel; danach wurde die Auswanderung immer mehr von der Binnenmigration in die heimischen Industrieregionen abgelöst. Der technische Fortschritt hatte in vieler Hinsicht unmittelbaren Einfluss auf die Wanderbewegungen: Mit der Geschwindigkeit und geringeren Gefährlichkeit des internationalen und transatlantischen Reisens durch die Erfindung des Dampfschiffs nahm auch die Zahl der Migranten erheblich zu. Es wuchs überdies die Zahl der Menschen aus Bayern, die den Radius ihrer früher interregionalen Arbeitswanderung oder ihrer Handelsreisen immer weiter ausdehnten. Auch die große Menge an Briefen, die allein zwischen den USA und Deutschland hin- und hergingen man schätzt sie auf 280 Millionen zwischen 1820 und 1914 -, ist ein Beleg dafür, dass die Verbesserung der Verkehrswege für den individuellen Kontakt zwischen dem Lokalen und der Welt von größter Bedeutung war. ${ }^{18}$ Alle Adressaten und ihre Verwandten oder Freunde, die in Bayern solche Briefe zu lesen bekamen, machten sich so ein Bild aus erster Hand über die USA, über die Lebensbedingungen, die Verdienstmöglichkeiten, die Preise. ${ }^{19}$ Die Bedeutung dieses veränderten Blicks der Daheimgebliebenen auf die Welt ist nicht hoch genug einzuschätzen: Hier löste die Migration der anderen bei den Zurückbleibenden und in ihrer Umgebung in Bayern Entwicklungen aus, die noch viel genauer untersucht werden müssten. ${ }^{20}$ Oft kam es infolge dieser Informationen aus der „Neuen Welt“ zu Kettenmigrationen ganzer Dörfer.

Die großen Auswanderungen des 19. Jahrhunderts führten auch aus Bayern zu $90 \%$ in die USA. ${ }^{21}$ Zwischen $1835 / 36$ und 1890 verließen über 600.000 Auswanderer Bayern, 22 über fünf Millionen das Gebiet des Deutschen Bundes und später des Deutschen Reichs. Zwischen 1846 und 1857 wanderten allein aus Bayern 140.000 Menschen in die USA aus, davon rund $45 \%$ aus der Pfalz, die bis weit ins 19. Jahrhundert jeweils den größten Anteil stellte. ${ }^{23} 17 \%$ stammten aus Unterfranken, $15 \%$ aus Oberfranken, $9 \%$ aus Mittelfranken, 5\% aus der Oberpfalz und nur $4 \%$ aus Schwaben, $3 \%$ aus Niederbayern und $2 \%$ aus Oberbayern. ${ }^{24}$ Neben dem Bevölkerungswachstum und den Hungerkrisen der vorangegangenen Jahre war es der Mangel an industriellen Arbeitsplätzen, der die Auswanderung antrieb. Für die deutlichen innerbayerischen Unterschiede sind die jeweilige Bevölkerungsdichte,

18 Helbich, Briefe 1988.

19 Fegert, Auswanderung ${ }^{2} 2014$.

20 Gestrich/Krauss, Zurückbleiben 2006.

21 Hamm/Henker/Brockhoff, Good Bye Bayern 2004; Bade/Oltmer, Deutschland 2007, S. 147; Hartmann/Schmid, Bayern 2011.

22 Statistisches Landesamt (Hrsg.), Bayern 1912.

23 Faltin, Auswanderung 1987.

24 Raithel, Auswanderung 2004, S. 26f., 34, Anm. 9. 
das Erbrecht sowie die frühere oder spätere Liberalisierung der ländlichen Welt zu nennen. Nach dem Ende des amerikanischen Bürgerkriegs zog es in der zweiten Hälfte der 1860er Jahre wieder viele Bayern in die USA, ebenso in dem Jahrzehnt nach 1880; die 170.000 Migranten und Migrantinnen aus Bayern dieser Welle stellten rund $10 \%$ der deutschen Amerikawanderer. Die politische Lage nach dem Hambacher Fest von 1832 und nach der gescheiterten Revolution von 1848 spielte, entgegen früheren Annahmen, keine quantitative Rolle bei der Auswanderung.

Für rund 5.000 Auswanderer aus Bayerisch-Schwaben ließ sich das Sozialprofil näher untersuchen: ${ }^{25}$ Sie stammten keineswegs aus übervölkerten, sondern eher aus den weniger industrialisierten Gegenden. Die Auswanderer, ob weiblich oder männlich, nahmen ein Durchschnittsvermögen von 200 bis 350 Gulden mit, waren also relativ wohlhabend; dies widerspricht der These, die Auswanderung habe vor allem als soziales Ventil gewirkt. Die Abschiebung von Besitzlosen kam zwar vor, fiel aber quantitativ kaum ins Gewicht. Bis 1860 wanderten fast zwei Drittel der bayerischen Schwaben im Familienverbund aus; erst danach gab es mehr junge und ledige Wanderer. Diese Auswanderer, so das Resümee, stammten aus einer liquiden Schicht, deren Mobilität möglicherweise darauf zurückzuführen ist, dass sie am Ende der Wanderung ein Leben in hergebrachten Wirtschaftsformen erhofften: mit einem eigenen Hof auf eigenem Grund und Boden. Auch für Oberfranken gilt, dass bis 1870 über $40 \%$ der erfassbaren männlichen Auswanderer aus dem landwirtschaftlichen Bereich kamen. ${ }^{26}$ Ein Großteil der Wandernden, für Oberfranken $64 \%$, war zwischen 16 und 40 Jahre alt; anfangs überwogen die Männer, zunehmend wanderten aber auch Frauen. Eine hohe vertikale, horizontale und schließlich geographische Mobilität war für die ländliche Gesellschaft Bayerns im 19. Jahrhundert nicht die Ausnahme, sondern die Norm. Die Auswanderung der nachgeborenen Söhne und Töchter nach Amerika erweist sich dabei vielfach als eine Frage des erweiterten Radius, nicht des grundsätzlichen Wandels von unterschichtlicher Migration. Im letzten Jahrhundertdrittel wuchs dann der Anteil der Auswandernden aus Handwerkerfamilien und der Fabrikarbeiter, die sich bessere Arbeitsbedingungen erhofften.

Wie viele solcher Auswanderer zurückkehrten oder, mit der zunehmenden Verbesserung der Verkehrsverbindungen, eine transatlantische Arbeitsmigration mit zwischenzeitlicher Rückkehr praktizierten, ist noch nicht erforscht. Für Deutschland insgesamt werden Rückwandererzahlen von etwa einem Viertel genannt - alles Menschen, die mit neuen Kenntnissen und Gewohnheiten, aber auch mit neuem technologischem Know-How in die alte Heimat zurückkehrten. ${ }^{27}$ Über ihre Geschäftsbeziehungen mit der alten Heimat, ihre Bedeutung für die Verbreitung aus-

25 Maidl, Auswanderung 1993; Knabe, Aufbruch 1992.

26 Schaub, Auswanderung 1989.

27 Vagts, Rückwanderung 1960. 
ländischer Patente, für Modernisierung und Mechanisierung weiß man wenig. Der lineare Blick auf Wanderungsprozesse versperrt den Blick auf solche Rückwanderungen: In der Vorstellung verlaufen diese immer von der alten in die neue Welt, vom Land in die Stadt, von der Tradition in die Moderne. Doch Migranten mussten sich mit den Regeln beider Systeme auskennen. Bei einer Rückkehr nach Bayern nahmen sie diese Kenntnisse wieder in die Ausgangsgesellschaft mit - oft ein wichtiger Faktor für Wissens- und Technologietransfer.

Die Migrationsforschung befasst sich vielfach mit „Migrationssystemen“ wie dem Sklavenhandel von (West-)Afrika nach Nordamerika oder eben der großen Auswanderung von Europa in die Vereinigten Staaten. ${ }^{28}$ Diese Migrationssysteme lassen sich auf der Weltkarte darstellen und zeigen die Bewegung von Menschen im geographischen Raum. Es gibt aber auch „kleine“ Wanderungssysteme, die für die Landesgeschichte von Bedeutung sind, wie die langen Traditionslinien saisonaler Arbeitswanderungen aus Norditalien und Tirol nach Bayern oder aus Polen ins Ruhrgebiet. Die Folgen der großen wie der kleinräumigen Wanderungen am regionalen oder lokalen Beispiel zu untersuchen, ist die Aufgabe der Landesgeschichte: Wie das Beispiel aus Bayerisch-Schwaben zeigt, sind auf diesem Wege manche Annahmen zu spezifizieren oder zu korrigieren.

\subsection{Binnenmigrationen und Arbeitszuwanderung}

Die große Auswanderung zog sich durch das gesamte 19. Jahrhundert, mit Höhepunkten Anfang der 1850er- und in den 1890er Jahren. Sie wurde jedoch zunehmend abgelöst durch die Wanderung der Arbeitsuchenden in die industrialisierten Regionen. Mit der Urbanisierung verlagerte sich das wirtschaftliche Schwergewicht vom agrarischen auf den gewerblichen und auf den Dienstleistungssektor mit neuen Sozialstrukturen und verstärkter sozialer und räumlicher Mobilität. Die Städte wandelten sich. Sie wuchsen um ein Vielfaches an, so Nürnberg von 91.000 Einwohnern 1875 auf 333.000 im Jahr 1910, München von 193.000 auf 596.000 Einwohner. Die entstehende kommunale Leistungsverwaltung musste die Folgen der Zuwanderung bewältigen..$^{29}$ Dabei ging es um Infrastruktur, um Trinkwasserversorgung, Abwasserentsorgung und Abfallbeseitigung, Energieversorgung, um Wohnungsbau, um neue öffentliche Gebäude, um Verkehr und Infrastruktur mit dem Bau von Bahn und Straßenbahn, um Kirchenbau, Versorgungseinrichtungen sowie um soziale Maßnahmen wie Armenpflege, Wohltätigkeit, Arbeitervereine oder die Einrichtung von Volksbädern. Nürnberg wurde früh zum Zentrum der bayerischen Industrialisierung; die erste deutsche Eisenbahn zwischen Nürnberg und Fürth war

28 Düwell, Migration 2006, S. 95-99.

29 Prinz/Krauss, München 1988, hier Glossar S. 378. 
hierfür Symbol und Startschuss. Das Verkehrswesen wurde Leitsektor der Industrie. Die Klett'sche Maschinenfabrik, von Theodor von Cramer-Klett zu einem Industrieimperium ausgebaut und später Grundlage der mächtigen MAN, soll dafür als Beispiel stehen. Aber auch die Elektroindustrie boomte. Im letzten Jahrhundertdrittel löste München zunehmend Nürnberg als wichtigstes bayerisches Industriezentrum ab. ${ }^{30}$ Augsburg und Schwaben blieben bis weit ins 20. Jahrhundert Zentren der Textilindustrie.

Es wanderten vor allem die mobilen und unternehmungslustigen jungen Menschen vom Land in die Stadt. Noch entstanden deshalb auf dem Land keine größeren Probleme, da der Geburtenüberschuss, auf Erfolge im Kampf gegen Hunger und Seuchen zurückzuführen, zu groß war. Es kam jedoch in manchen Gegenden bereits zu Entvölkerung und die Gewichte begannen sich zugunsten des urbanen Raumes zu verschieben. Dieser Prozess setzte sich mit Unterbrechungen und mit einer vehementen Beschleunigung in den 1950er Jahren fort: Mit der Mechanisierung und Technisierung der Landwirtschaft erlebte Bayern den endgültigen Wandel von einem Agrarstaat zu einer modernen Dienstleistungsgesellschaft. Gemessen wird diese Veränderung an der Landflucht und damit an der Zahl der in den Städten lebenden Menschen.

Bereits um 1900 reichten jedoch die Arbeitskräfte, die das eigene Land hervorbrachte, nicht aus: Seit dieser Zeit wandelte sich Bayern von einem Arbeitskräfteausfuhrland zu einem Arbeitskräfteeinfuhrland. Um die Jahrhundertwende arbeiteten im gesamten Deutschen Reich rund 70.000 Italiener und Italienerinnen, dies vor allem in Bayern, Baden und Württemberg. In Bayern kamen die ausländischen Arbeitswilligen häufig aus Norditalien - als Erntehelfer, Bahnbauarbeiter, Ziegeleiarbeiter; Frauen arbeiteten auch in der Textilindustrie. ${ }^{31}$ Vielfach handelte es sich um Saisonarbeiter. So fuhren aus Bayern $60 \%$ der dort arbeitenden Italiener im Winter nach Hause, aus Baden nur 33\%.

Man brauchte viele Kräfte: In Münchner Vororten mit Ziegelindustrie, wie in Berg am Laim, lebten in den Sommermonaten oft 900 italienische Ziegelarbeiter neben 1350 Einheimischen, in Oberföhring waren es um 1900 rund 900 Einwohner und im Sommer zusätzlich 600 bis 800 Gastarbeiter. ${ }^{32}$ Im Ersten Weltkrieg siedelten sich dann große Rüstungsunternehmen in Bayern an, was zu einer Zuwanderung tausender Industriearbeiter in das Umfeld der großen Städte führte.

Im Zweiten Weltkrieg warb man erst italienische Gastarbeiter an, deren Arbeitsbedingungen anfangs von der "Deutschen Arbeitsfront“ mit der faschistischen Schwesterorganisation in Italien ausgehandelt wurden. Bald hörte man jedoch in Bayern alle Sprachen Europas: Aus besetzten Ländern wurden Arbeiter rekrutiert,

30 Krauss, Kommerzienräte 2016.

31 Fabbro, Transalpini 1996.

32 Kasberger, Lehm 2007. 
die man zunächst mit Versprechungen, später immer mehr mit Gewalt ins Reich holte. Auch kriegsgefangene Franzosen wurden zur Arbeit eingesetzt. Als Arbeitgeber fungierten Rüstungsbetriebe, aber auch die Reichsbahn, Familien, die Landwirtschaft, der Straßenbau. Die Bayerischen Motorenwerke und vergleichbare Betriebe profitierten besonders von der Arbeit ausländischer Zwangsarbeiter. ${ }^{33}$ Die sogenannten Ostarbeiter, also Russen, Ukrainer etc., standen am untersten Ende der Hierarchie, sie wurden schlecht bezahlt, kaserniert, mit einem „O“ oder „SU“ (für Sowjetunion) auf der Kleidung gekennzeichnet, von der Gestapo überwacht und bei kleinsten Vergehen streng bestraft. Ende September 1944 waren allein im Arbeitsamtsbezirk München und Oberbayern rund 80.000 Männer und knapp 42.000 Frauen als ausländische Zivilarbeiter beschäftigt, $37 \%$ stammten aus der Sowjetunion, 18,2\% aus Polen, 17,2\% aus Frankreich.

Als diese Arbeiter bei Kriegsende wegfielen, deckten zunächst die Vertriebenen und Flüchtlinge den Bedarf an Arbeitskraft. Spätestens seit Beginn der 1960er Jahre, nachdem 1961 der Mauerbau den weiteren Zustrom von Menschen aus der DDR abgeschnitten hatte, beschleunigte sich die Anwerbung von "Gastarbeitern“ aus Italien und anderen europäischen Ländern: Bereits 1955 wurde das deutschitalienische Anwerbeabkommen unterschrieben, nur zehn Jahre nach dem Ende italienischer Zwangsarbeit in Deutschland. Mit Beginn der 1960er Jahre stieg der Bedarf an Arbeitskräften weiter an; arbeiteten 1961 noch 1,5\% Ausländer in Schwaben, so waren es 1970 schon 5,9\%. 1960 wurden Anwerbeabkommen mit Spanien und Griechenland abgeschlossen, 1961 mit der Türkei, 1963 mit Marokko und Südkorea, 1964 mit Portugal, 1965 mit Tunesien, 1968 mit Jugoslawien. Der Anteil der Italiener an den ausländischen Arbeitskräften lag in Bayern anfangs hoch, 1960 waren es 14.000 Italiener und Italienerinnen sowie 300 Türken; 1971 standen 51.000 Italienern bereits 71.000 Türken und 88.000 Jugoslawen gegenüber. ${ }^{34}$

Die ausländischen Arbeitnehmer arbeiteten zu Beginn oft in der Baubranche oder in der Industrie zu Dumping-Löhnen. Italiener und andere Angeworbene aus EG-Ländern hatten eine unbeschränkte Arbeitserlaubnis; sonst gab es nur EinJahres-Verträge. Die Niederlassung der Arbeiter in Deutschland war nicht geplant, „Deutschland ist kein Einwanderungsland“, lautete die Parole. Doch es holten immer mehr Arbeiter ihre Familien nach. Seit 1971 gab es die Möglichkeit von FünfJahres-Verträgen. Nach der „Ölkrise“ und einer Rezession folgte 1973 der Anwerbestopp. 1974 lebten in Bayern 653.000 Ausländer, das waren 6\% der Bevölkerung. Nun bemühte man sich mehr um ihre Integration. Es kam zu neuen sozialen Hierarchien: Mit der Ankunft einer neuen Gruppe stiegen jeweils die anderen auf; als Beispiel für den Aufstieg gelten Restauranteröffnungen.

33 Grossmann, Zwangsarbeiter 1986; Bösl/Kramer/Linsinger, Gesichter 2005.

34 Krauss, Arbeitswanderer 2010. 
Industrialisierung und Urbanisierung - so lässt sich resümieren - waren eng mit Migration verbunden. Die Migranten kamen zunächst noch aus dem engeren Umland, im Zuge der Hochindustrialisierung wuchs der Radius dieser Arbeitswanderung jedoch immer mehr. Man rief Arbeitskräfte, doch es kamen Menschen, deren Anwesenheit die Aufnahmegesellschaft veränderte, sie durchmischte und die Einheimischen dazu veranlasste, sich immer wieder neu ihrer selbst zu versichern. Jede neue Zuwanderergruppe unterschichtete die vorhergehende und trug $\mathrm{zu}$ ihrem sozialen Aufstieg bei. Die Kinder und Kindeskinder der Zuwanderer waren dann bereits selbst Einheimische.

\subsection{Migrationen nach 1933 und nach dem Zweiten Weltkrieg: Exil, Flucht und Vertreibung}

Mit der Machtübernahme der Nationalsozialisten begann auch in Bayern der Exodus der Menschen, die aus politischen, religiösen oder rassistischen Gründen verfolgt wurden. Bereits nach dem Reichstagsbrand im Februar 1933 verließen besonders gefährdete Sozialdemokraten, Kommunisten und andere politische Gegner der Nationalsozialisten Bayern; weitere folgten im Laufe des Jahres. Zunächst flohen die politisch Verfolgten vornehmlich in die Nachbarländer Österreich, Schweiz, Tschechoslowakei, Italien, die Niederlande, Skandinavien oder über das Saargebiet nach Frankreich. Einige, wie der SPD-Abgeordnete Waldemar von Knoeringen, versuchten vom Exil aus den Widerstand in Bayern $\mathrm{zu}$ organisieren. Im Laufe der 1930er Jahre emigrierten immer mehr Juden oder Menschen mit jüdischer Abstammung, die letztlich weit über $90 \%$ der Emigration ausmachten. ${ }^{35}$ Viele zögerten die Auswanderung $\mathrm{zu}$ lange hinaus, hofften sie doch, dass sich die antisemitischen Attacken des Regimes legen würden. Doch nach den „Nürnberger Rassegesetzen“ von 1935 und der Reichspogromnacht von 1938 wurde die Bedrohung auch für diejenigen zunehmend deutlicher, die bisher noch auf ein Abflauen der Verfolgung gehofft hatten. Die zunehmende Ausraubung der jüdischen Minderheit durch „Arisierung“ des Besitzes, durch „Reichsfluchtsteuer“ und andere Abgaben ließ den Verfolgten immer weniger Spielräume. Außerdem schlossen die meisten Zielländer unter Verweis auf eigene wirtschaftliche Probleme und Arbeitslosigkeit, aus Furcht vor Überfremdung oder wegen antisemitischer Positionen ihre Grenzen. Aus dem deutschsprachigen Raum flohen insgesamt rund 500.000 Menschen, davon etwa 30.000 wegen ihrer politischen Haltung, alle anderen wurden aus rassistischen Gründen verfolgt; etwa 360.000 kamen aus dem Deutschen Reich, 140.000 aus Österreich. Genauere Zahlen sind schwer zu finden. Aus München emigrierten zwischen 1933 und 1938 bereits 3.574 Personen, davon 701 nach Palästina und 627 in die USA. Wer

35 Krauss, Heimkehr 2001. 
es nicht mehr schaffte, das Land zu verlassen, erlitt Entrechtung und Verfolgung, oft auch Deportation und Tod. Nur wenige der Emigrierten kehrten nach 1945 in die alte Heimat zurück. Darunter stellen die politischen Emigranten die größte Gruppe.

In der Nachkriegszeit des Zweiten Weltkriegs vergrößerte sich Bayerns Einwohnerzahl durch den Zustrom von Heimatvertriebenen aus den ehemals deutschen Ostgebieten und aus dem Sudetenland sowie später von Flüchtlingen aus der SBZ/ DDR um ein Fünftel bis ein Viertel - das waren rund 1,8 Millionen Menschen, darunter fast eine Million aus den ehemaligen Sudetengebieten. ${ }^{36}$ Die Vertriebenen kamen nicht freiwillig: Es handelte sich um eine politisch bedingte Zwangsmigration. Ihre Aufnahme und Eingliederung stellte eine der größten Herausforderungen dieser Jahre dar.

Während des Krieges waren wegen der Bombengefahr viele Menschen aus den großen Städten des Deutschen Reiches aufs Land evakuiert worden. 1946 lebten allein in Bayern über 410.000 außerbayerische Evakuierte, Ende 1948 immer noch 268.000. Mit Kriegsende waren auch Zwangsarbeiter, Kriegsgefangene und Insassen von Konzentrationslagern befreit worden. Ein Großteil der "displaced persons" strebte die Auswanderung an; allein Oberbayern wurde zwischen 1945 und 1951 zur Durchgangsstation für 120.000 auswandernde Juden. Bayern war gewissermaßen „Wartesaal“ auf dem Weg in die Auswanderung, die größtenteils nach Palästina führte. ${ }^{37}$

Zunächst sahen die amerikanischen Besatzer die Aussiedlung und Vertreibung Deutscher aus dem Osten als Transportproblem an. Dies hielten sie für lösbar; es sollte nur die „humane Durchführung“ gewährleistet sein. Offiziell galt das Flüchtlingsproblem als „rein deutsche Frage“, doch die Besatzer waren von Beginn an eng in die Bewältigung des Zuzugs eingebunden und bestimmten die Grundlinien mit. Im Juli 1945 erfuhren die deutschen Stellen, dass eine solche Abschiebung geplant sei. Anfangs glaubte man, das Rote Kreuz werde die Erstbetreuung übernehmen können. Seit dem Sommer 1945 etablierten sich dann in den wieder entstehenden Ländern bis hinunter auf die Ebene der Landkreise mit speziellen Vollmachten ausgestattete Flüchtlings-Sonderverwaltungen, die Flüchtlinge auch in Privatquartiere einweisen konnten. Daneben wurden auch bestehende oder schnell neu errichtete Lager und Massenquartiere zur Unterbringung herangezogen. Selbsthilfeorganisationen ließen die Besatzer nicht zu, da sie fürchteten, es könnte so ein Minderheitenproblem entstehen. Daher wollten sie auch die geschlossene Ansiedlung von Dorfgemeinschaften unterbinden.

Bei den ersten demokratischen Wahlen auf Kreis- und Länderebene konnten die Vertriebenen meist noch nicht mitwählen. An der Beratung des Flüchtlingsgesetzes wurden die Neuankömmlinge jedoch in Bayern auf ausdrücklichen Wunsch des aus

36 Krauss, Integrationen 2008.

37 Wetzel/Königseder, Lebensmut 1994. 
dem Exil zurückgekehrten Ministerpräsidenten Wilhelm Hoegner (SPD) beteiligt. Das im Februar 1947 in Bayern erlassene „Gesetz über die Aufnahme und Eingliederung deutscher Flüchtlinge vom 19.2.1947“ legte die demokratische Mitwirkung der Vertriebenen und Flüchtlinge sowie eine Beschäftigungsquote im öffentlichen Dienst fest. Nach der Aufhebung des Verbotes, sich in Parteien zusammenzuschlieBen, entstand 1950 mit dem „Bund der Heimatvertriebenen und Entrechteten“ (BHE) eine Flüchtlingspartei, die beträchtliche Wahlerfolge verbuchen konnte.

Zunächst stand die wirtschaftliche Integration im Mittelpunkt der Bemühungen. Da die Freizügigkeit aufgehoben war, mussten die Vertriebenen häufig auf dem Land ausharren und sich um Arbeit in der Landwirtschaft bemühen. Erst nach der Gründung der beiden deutschen Staaten gab es eine Möglichkeit der legalen Umsiedlung. Auf der Suche nach Arbeit und Verdienst zog es über eine Million Flüchtlinge und Vertriebene, darunter viele aus Bayern, in die Industriereviere NordrheinWestfalens. Ein weiteres wichtiges Anliegen war es, Familie, Freunde und Nachbarn wiederzufinden.

Besonders schwierig war der Neuanfang für diejenigen, die aus den arbeitsteilig organisierten Spezialindustrien des Sudetenlandes stammten; ${ }^{38}$ dazu gehörten u.a. die Gablonzer Glas- und Schmuckindustrie, die Haida-Steinschönauer Glasindustrie, die Schönbacher und Graslitzer Musikinstrumentenherstellung. Die Gablonzer schafften eine zumindest in Teilen gemeinsame Neuansiedlung bei Kaufbeuren und Marktoberdorf im Allgäu und konnten umfängliche Kreditmittel einwerben. Neben dem Hauptansiedlungsgebiet mit über 520 Betrieben gab es aber auch Unternehmen der Gablonzer Industrie u.a. in den bayerischen Landkreisen Bayreuth, Oberallgäu, Günzburg, Augsburg, Mühldorf am Inn und Ansbach. Andere Industrien waren nicht so erfolgreich wie die Gablonzer, aber immerhin erreichten einige der Spezialindustrien zumindest eine teilweise Neuansiedlung. Die Firmengründungen trugen zur Industrialisierung des bayerischen Landes bei. Sie waren jedoch den Krisen der 1970er Jahre und der Billigkonkurrenz aus Asien kaum gewachsen.

Es gab vier Schritte der Integration: Notdürftige Aufnahme - vorläufige Unterbringung - endgültige Sesshaftmachung mit wirtschaftlicher Integration. Als vierter Schritt folgte seit Ende der 1940er Jahre die kulturelle Integration. Diese bezog gleichermaßen die Erinnerung an die alte Heimat und die Aneignung der neuen mit ein. Die Vertriebenen brachten großen Bildungshunger und hohe Aufstiegsmotivation mit, ebenso die Erfahrung gut ausgestatteter Volksbibliotheken und funktionierender Volksbildungseinrichtungen. Aus landsmannschaftlichen Zusammenschlüssen erwuchs dann neues Selbstbewusstsein.

Die bayerische Aufnahmegesellschaft, auf die diese Migranten stießen, war keineswegs homogen. Gerade in dieser Situation schlossen sich Einheimische dann oft zu „Wir-Gruppen“ zusammen, um sich gegen „die Fremden“ zur Wehr zu setzen.

38 Prinz, Integration 1984. 
Man wolle „Herr im eigenen Haus bleiben“, schrieb ein Flüchtlingskommissar. Es ging dabei nicht zuletzt um einen Machtkampf zwischen den Alteingesessenen und den Hinzukommenden, einen Kampf um die knappen Ressourcen, um Einfluss und Deutungshoheit. Stand zunächst noch unmittelbar der sehr materielle Streit um Essen und Unterbringung im Mittelpunkt, so bezog sich das bald auch auf andere Lebensbereiche: So reaktivierten die Einheimischen in den Dörfern angeblich ,althergebrachte" Traditionen, um sich gegen die Wünsche der aus industrialisierten Gebieten stammenden Vertriebenen zur Wehr zu setzen. Obwohl sich die Konflikte der unmittelbaren Nachkriegszeit auch dank des wirtschaftlichen Aufschwungs abschwächten, blieben Exklusionsmechanismen und Vorurteile lange bestehen.

Es gab nicht nur die Zuwanderung von Vertriebenen. Nach 1945 profitierte Bayern auch von der deutschen Teilung, so durch den Zuzug von Großforschungseinrichtungen wie der Max-Planck- oder der Fraunhofer-Gesellschaft. Einzelne MaxPlanck-Institute, wie das Institut für Silikatforschung in Würzburg, konnten dank kommunaler Bemühungen in Franken angesiedelt werden. ${ }^{39}$ Es verlegten aber auch bedeutende Wirtschaftsunternehmen, wie die Allianz, ihren Sitz nach Bayern, ebenso große Leipziger Verlage. ${ }^{40}$ Erlangen profitierte in besonderem Maße von dieser Bewegung: Die Verlegung der Zentrale des Konzerns Siemens von Berlin nach Erlangen war für die Stadt ein zentraler Wachstumsfaktor. Die Zuwanderungen legten die Grundlagen für die innovative technologische Forschungslandschaft in Bayern. Im Wirtschaftsbereich war Migration, so ist dies zu resümieren, für Bayern sowohl mit Blick auf qualifizierte Arbeitskräfte wie auf Unternehmer und Unternehmen eine zentrale Voraussetzung des späteren wirtschaftlichen Erfolgs.

Die Zuwanderung unterschiedlicher Gruppen politisch und wirtschaftlich in ihren Heimatländern bedrängter Personen hat das Thema Migration wieder auf die zentrale Agenda gesetzt. Kamen die russlanddeutschen Zuwanderer in den 1990er Jahren noch zumindest mit der Fiktion deutscher Abstammung, so galt und gilt dies nicht für Bürgerkriegsflüchtlinge und Asylsuchende. Längst ist Migration zu einem hochpolitisierten Thema geworden und die Fragen der Zuwanderung spalten die Gesellschaft. Die Angst vor Migration tritt dabei deutlich zutage: Es ist die Angst vor vermuteter Konkurrenz und Überfremdung, die in Bayern durch die Politik besonders bedient wird. Dies macht erneut deutlich, wie sehr die scheinbare Homogenität der längst vielfach durchmischten Gesellschaft in Bayern gegen jedes bessere Wissen behauptet wird.

39 Deutinger, Agrarland 2001.

40 Krauss, Nachkriegskultur 1985. 


\subsection{Resümee}

Die Erforschung von Migrationen erfordert per se einen regionalen Zugriff, sei es auf die Ausgangs- oder die Aufnahmegesellschaft; dies zeigen auch die Beiträge in der „Enzyklopädie Migration in Europa seit dem 17. Jahrhundert“, die einen Großteil der bisher im europäischen Raum untersuchten Migrationsbewegungen in knappen Überblicksdarstellungen bündelt. ${ }^{41}$ Die betrachteten Räume und Gruppen sind dabei von unterschiedlicher Größe. Doch die dahinterstehenden Forschungsarbeiten beziehen sich oft auf Regionalstudien.

Der regional- und landesgeschichtliche Zugriff auf das Thema Migration lässt sich besonders fruchtbar machen, wenn er unter vergleichendem Blickwinkel geschieht: Erst hier zeigen sich Unterschiede und Gemeinsamkeiten. Ein Beispiel dafür ist der Band „Integrationen. Vertriebene in den deutschen Ländern nach 1945“, der sich eben diesem Ansatz verpflichtet sieht. ${ }^{42}$ Diachron vergleichende Arbeiten zur Migration, wie sie im Rahmen des ,Stuttgarter Arbeitskreises für Historische Migrationsforschung " entstanden sind, ${ }^{43}$ machen ebenfalls deutlich, wie sehr Migrationsstudien von regionalen Zugängen leben. Für die Bayerische Landesgeschichte sieht man am Beispiel des sicherlich am intensivsten auf lokaler Ebene erforschten Themas der Integration von Vertriebenen und Flüchtlingen die Bedeutung der vergleichenden regionalen Einordnung solcher Studien für einen weiterreichenden Erkenntnisgewinn.

\section{Brandenburg}

\section{Ulrich Niggemann}

Die Mark Brandenburg - bestehend aus der Altmark, der Mittelmark, aber auch der Uckermark und der Neumark - war seit dem Mittelalter von Migrationsvorgängen geprägt. Im Gegensatz zu manch anderer Region spielt Migration bzw. Kolonisation hier in der Traditionsbildung und im kollektiven Gedächtnis eine wichtige Rolle, sei es im Gründungsmythos einer „Landnahme“ oder sei es im Rahmen des Erfolgsnarrativs der „Hohenzollernschen Kolonisationen“ des 17. und 18. Jahrhunderts. Beide Narrative können mit guten Gründen problematisiert werden, ohne damit die Bedeutung von Migration für die Ausformung der Region in politischer, ökonomischer und kultureller Hinsicht zu relativieren. Der folgende Beitrag kann dies größ-

41 Bade, Enzyklopädie 2007.

42 Krauss, Integrationen 2008.

43 Gestrich/Krauss, Zurückbleiben 2006; Gestrich/Krauss, Migration 1998; Krauss/Sonnabend, Frauen 2001. 
tenteils nur andeuten, will aber in einem an der Chronologie orientierten Zugriff zumindest einige der grundlegenden Entwicklungen knapp skizzieren.

\subsection{Siedlungsmigration in der Mark Brandenburg bis zum Beginn der Neuzeit}

Die Besiedlung und Erschließung von Landschaften und Räumen steht immer in Zusammenhang mit Migrationsprozessen. Dementsprechend lassen sich die frühesten Siedlungsspuren in dem Gebiet zwischen Elbe und Oder entlang der Havel und der Spree als Spuren von Wanderungsbewegungen lesen. Wohl etwa seit dem 7. Jahrhundert ist von einer Besiedlung durch slawisch sprechende Verbände auszugehen. Im 10. Jahrhundert drangen vom Westen her verstärkt Sachsen militärisch in den Raum bis zur Oder vor. Sie gründeten die Bistümer Brandenburg und Havelberg und bemühten sich um die Christianisierung der slawischen Bevölkerung. Die damit einhergehenden Siedlungsversuche vonseiten des Ostfränkischen Reiches waren zunächst nicht von Dauer. Erst seit dem 12. Jahrhundert begann mit der Etablierung der Markgrafschaft Brandenburg die intensivere herrschaftliche Durchdringung. Man sollte sich freilich davor hüten, das Land vor der ,Landnahme“ durch sächsische oder ostfränkische Christen als weitgehend unbewohnt darzustellen. Selbst vor der slawischen Besiedlung dürften hier bereits Menschen gelebt haben. Der Mythos von einem leeren und wilden Land, das gewissermaßen bereitlag für die ,Landnahme', ist eine Vorstellung, die mit vielen Siedlungs- und Kolonisationsvorgängen verbunden ist und die vielfach auf spezifischen Wahrnehmungsmustern, aber auch intentionalen Ausblendungen bereits vorhandener Bevölkerungsgruppen beruht. Dennoch ist es sicher richtig, wenn man konstatiert, dass die Mark Brandenburg (mit Ausnahme der Altmark) jenseits des deutschsprachigen Altsiedellandes lag und dass sie in ihrer historischen Entwicklung wesentlich von den Siedlungsbewegungen des Mittelalters geprägt war. Damit steckt per se ein migratorisches Moment in der Geschichte dieser Landschaft. ${ }^{44}$

Mit der Etablierung der Askanier in der Mark Brandenburg seit 1134 begann eine auch obrigkeitlich gelenkte Immigration, die insbesondere der Herrschaftssicherung diente. Nachdem Albrecht von Ballenstedt („Albrecht der Bär“, 1134-1170) mit der sächsischen Nordmark (der späteren Altmark) belehnt worden war, griff er bald in die Gebiete östlich der Elbe aus und setzte sich dort fest. Als zentrale Burganlagen errichtete er die Brandenburg und Spandau. Offenbar wurden zur Herrschaftssicherung und zur Erschließung des Landes bereits in dieser Phase gezielt Siedler aus dem Rhein- und Maasgebiet angeworben. Auch Holländer, Seeländer und Flamen

44 Hahn, Geschichte 2009, S. 11-13; Heinrich, Geschichte 1981, S. 27f.; Asche/Niggemann, Land 2015. 
ließen sich in der Mark Brandenburg nieder, die als neue, loyale Untertanen die Herrschaftsbildung des Markgrafen unterstützten. Klostergründungen, die ebenfalls gezielt von den Askaniern gefördert wurden, bildeten zugleich Zentren der fortschreitenden Christianisierung. Um sie herum entstanden Rodungen und Niederlassungen, die das Land strukturierten und administrativ durchdrangen. Ähnliches gilt für Adelssitze, die ebenfalls Siedler anzogen. ${ }^{45}$

Mit dem Aussterben der männlichen Linie der brandenburgischen Askanier 1320 endete zunächst die Phase der territorialen Durchdringung und der aktiven Besiedlungspolitik. Unter den Wittelsbachern und den Luxemburgern, die zeitweise die Markgrafschaft regierten, fehlte es vielfach an herrschaftlicher Präsenz vor Ort, sodass adelige Grundherren und die Städte ihre eigene Machtbasis ausdehnen konnten. Als 1411 der Burggraf von Nürnberg, Friedrich VI. von Hohenzollern (14151471), mit der Mark belehnt wurde, mussten er und seine Nachkommen zunächst ihre Ansprüche gegen den Adel und gegen die Städte durchsetzen. Um dies zu erreichen, brachten sie auch Gefolgsleute aus dem fränkischen Raum mit in die Mark.46 Hier kam es also in quantitativ sehr geringem Umfang zu einer Elitenmigration, zur Niederlassung von Adeligen und Heeresangehörigen in der Mark.

\subsection{Ausweisung, Vertreibung, Krieg: Emigration aus Brandenburg}

Die beginnende Neuzeit war indes zunächst auch eine Phase der Bevölkerungsverluste. In diesen Zusammenhang gehören etwa die gezielten Vertreibungen und Ausweisungen nicht erwünschter Minderheiten im Zuge frühmoderner Staatsbildungsprozesse. Betroffen waren davon einerseits "Zigeuner" (d.h. Sinti und Roma, aber auch andere mobile Minderheiten) sowie andererseits die Juden. RomaGruppen tauchten wohl bereits zu Anfang des 15. Jahrhunderts in der Mark Brandenburg auf. Obwohl sie von Beginn an Stigmatisierungsprozessen ausgesetzt waren, wurden sie erst allmählich zu Opfern einer immer rigoroseren Ausweisungspolitik, die sich vom beginnenden 16 . bis ins 18 . Jahrhundert hinein massiv steigerte. Das ist nicht typisch für Brandenburg, sondern eine Entwicklung, die fast überall im werdenden frühmodernen Flächenstaat beobachtet werden kann. Mit der Verfestigung von Grenzen und territorialer Hoheit wurde zunehmend versucht, bestimmten Formen grenzüberschreitender Mobilität einen Riegel vorzuschieben und eine Norm der Sesshaftigkeit durchzusetzen. Die Einreiseverbote, oft verbunden mit drakonischen Strafandrohungen, hatten jedoch zur Folge, dass ein legaler Aufenthalt

45 Hahn, Geschichte 2009, S. 15; Assing, Landesherrschaft 1995, S. 102-109; Partenheimer, Albrecht 2011, S. 143-145.

46 Assing, Landesherrschaft 1995, S. 132-168; Böcker, Festigung 1995; Hahn, Geschichte 2009, S. 34-36. 
von Roma-Gruppen fast nirgendwo mehr möglich war. Umherziehen wurde somit auch $\mathrm{zu}$ einer Überlebensnische, die zum Teil gerade erst durch die massiven Verbote erzwungen wurde, wodurch sich erst eine spezifische Kultur der permanenten Mobilität herausbildete, die aber eben auch erneute und verstärkte Diskriminierung zur Folge hatte. ${ }^{47}$

Etwas anders sah es bei der religiösen Minderheit der Juden aus. Jüdische Gemeinden gab es in der Mark schon seit dem Mittelalter. Vermutlich kamen sie bereits mit den Kolonisationen der Askanier, nachweisbar sind sie - etwa in Berlin seit dem späten 13. Jahrhundert. Schon unter Kurfürst Friedrich II. kam es $1446 \mathrm{zu}$ einer ersten Vertreibung, deren genaue Ursachen unbekannt sind, die aber bereits ein Jahr später, 1447, in eine Wiederaufnahme mündete. Jüdisches Leben in Brandenburg blieb aber - wie fast überall - prekär und von Diskriminierung, Ausgrenzung und Anfeindung geprägt. Bis auf eine dünne jüdische Oberschicht, die vor allem im Handel und Finanzwesen tätig war, blieb die größere Zahl der Juden auf Klein- und Hausierhandel beschränkt. Im 16. Jahrhundert setzten dann mehrere Wellen der Verfolgung und Ausweisung ein. Die erste stand im Zusammenhang mit einem Hostienschändungsprozess, im Zuge dessen im Jahr 1510 etwa 60 Juden ausgewiesen wurden und zahlreiche weitere außer Landes flüchteten. Eine umfassende Ausweisung - auf „ewige Zeiten“, wie es im Ausweisungsedikt hieß - fand 1571 statt, und zwar erneut als Folge eines Prozesses, dieses Mal gegen den Münzmeister Lippold Ben Chluchim, dem vorgeworfen worden war, sich unrechtmäßig bereichert zu haben. Hintergrund waren anscheinend die Schulden, die Kurfürst Joachim II. Hektor bei ihm hatte und die sein Sohn, Kurfürst Johann Georg, bei seinem Regierungsantritt 1571 nicht übernehmen wollte. ${ }^{48}$ Für hundert Jahre endete damit die legale Existenz des Judentums in der Mark Brandenburg.

Es war indes erst der Dreißigjährige Krieg, der eine tiefe Zäsur in der Demographiegeschichte darstellte. Die Auswertung der Landreiterberichte, die ab 1652 in verschiedenen Landesteilen erstellt wurden, zeigt, dass die ländlichen Gebiete der Kurmark Brandenburg zu den am stärksten kriegszerstörten Regionen des Reiches gehörten. Hunger, Seuchen und - in geringerem Ausmaß - die Kriegshandlungen selbst führten zu hohen Sterblichkeitsraten. Darüber hinaus war es aber auch die Emigration, die wesentlichen Anteil an den Bevölkerungsverlusten hatte. Wiederholte Truppendurchzüge und hohe Kontributionsforderungen der durchmarschierenden Heere hatten zum Niedergang zahlreicher Höfe und Dörfer geführt. Tausende von Menschen flohen vor den heranrückenden Soldaten oder verließen nach den Durchzügen ihre ruinierten Hofstellen. Insgesamt dürfte die Mark Brandenburg bis zur Hälfte ihrer Bevölkerung verloren haben, in einigen Gebieten - etwa der

47 Härter, Grenzen 2016; Hehemann, Sinti 1992.

48 Scheiger, Juden 1990, S. 159-164. 
Uckermark - ist gar von $90 \%$ Bevölkerungsverlusten die Rede. ${ }^{49}$ Somit sind auch die demographischen Auswirkungen des Krieges Untersuchungsgegenstand einer regionalhistorisch orientierten Migrationsforschung, die sich nicht nur mit Einwanderungsbewegungen, sondern auch mit der Abwanderung aus der Region und ihren Folgen zu beschäftigen hat.

\subsection{Landesausbau und merkantilistische Bevölkerungspolitik}

Parallel zu den Emigrationen des 16. Jahrhunderts gab es indes auch Einwanderungsbewegungen. Bereits unter Kurfürst Joachim II. (1535-1571) siedelten sich erstmals kleinere Gruppen reformierter Niederländer in der Prignitz und in der Stadt Stendal an, wo sie insbesondere in der Textilverarbeitung tätig wurden. Doch vor allem nach den verheerenden Zerstörungen und Bevölkerungsverlusten durch den Dreißigjährigen Krieg setzten verstärkt Wanderungsbewegungen aus den kriegsverschonten Gebieten mit Bevölkerungsüberschuss ein. Dabei ist zu bedenken, dass es sich nicht um einen simplen Einwanderungsvorgang handelte, sondern um ein sehr komplexes Migrationsgeschehen. Auf der Suche nach Chancen, nach Hofstellen und besseren rechtlichen Bedingungen zogen Menschen aus den weniger verwüsteten Gebieten Holsteins, Lüneburgs, Braunschweigs und Mecklenburgs in die Mark Brandenburg. Zugleich hielten aber auch Abwanderungen noch lange nach dem Krieg an, und auch manch eine Neuansiedlung war keineswegs von Dauer. Gründe dafür waren die schwierige Ausgangslage nach dem Krieg, aber auch die Tendenz insbesondere der Gutsbesitzer, durch ,Bauernlegen', also den Einzug von bäuerlichen Stellen, die Eigenwirtschaft zu vergrößern. Hinzu kam die Verschärfung der personenrechtlichen Bindungen nach dem Krieg. Um die Menschen auf den Hofstellen $\mathrm{zu}$ halten, führten die adeligen Gutsherren eine strikte Erbuntertänigkeit ein und führten geflohene Leibeigene zum Teil mit Gewalt auf ihre Stellen zurück. Nur auf den Domänen sah die Situation für die Bauern etwas besser aus. ${ }^{50}$

Die Regierung unter Kurfürst Friedrich Wilhelm (1640-1688) versuchte, diese kontinuierliche Abwanderung durch Abwerbungsverbote zu unterbinden und zugleich den Zuzug zu forcieren, indem sie verschiedene Anreize für die Niederlassung bot. Den Hintergrund dieser gezielten Bevölkerungspolitik bildete der kameralistische Diskurs, der keineswegs nur das gelehrte Denken prägte, sondern auch die praktische Politik. Unter dem Begriff des ,Kameralismus‘ sind zahlreiche Maßnahmen der ,guten Policey` zu verstehen, zu denen neben Aspekten der Staatsverwaltung insbesondere auch die Steuerpolitik, die umfassende (zumeist stark dirigistische) Förderung von Handel und Gewerbe, der Landesausbau sowie die Bevölke-

49 Asche, Neusiedler 2006, S. 40-54.

50 Ebd. S. 115-128, 143-375; Niggemann, Peuplierung 2016, S. 176-182. 
rungspolitik gehörten. Es ist ein typisches Merkmal kameralistischer Politik, dass sich Regierungsmaßnahmen erstmals in größerem Umfang und in systematischer Weise auf die Ökonomie als ein Feld der praktischen Politik bezogen. Im Rahmen einer umfassenden Wirtschaftspolitik kam dem Faktor Bevölkerung durchaus einige Bedeutung zu. Von der Bevölkerungsvermehrung versprachen sich die Obrigkeiten eine Erhöhung der Produktion und des Konsums, was die Hebung des allgemeinen Wohlstands und damit auch der Steuerleistung zur Folge haben sollte. Neusiedler und Kolonisten zu gewinnen, wurde als Mittel zur Verbesserung der wirtschaftlichen Verhältnisse wie auch der Staatseinnahmen daher von einigen Kameralisten explizit empfohlen. Der Mensch wurde somit zu einer wirtschaftlichen Ressource, die staatlicher Planung unterworfen wurde. ${ }^{51}$

Nach dem Dreißigjährigen Krieg spielten kameralistische Doktrinen auch in Brandenburg eine erhebliche Rolle. Dies schlug sich in allgemeinen Einwanderungsaufforderungen nieder, und darüber hinaus bemühte sich der Kurfürst auch direkt um die Anwerbung besonders erwünschter Gruppen. Seit 1646 wurden mithilfe niederländischer Siedler ländliche Kolonien angelegt und wüst gefallene Dörfer wiederbesiedelt. Insbesondere im havelländischen Kreis sowie ab 1649 und 1652 in den Ämtern Zehdenick, Liebenwalde, Fehrbellin, Chorin, Tangermünde und Gramzow entstanden auf diese Weise Niederlassungen. Verschiedentlich gingen diese Wiederbesiedlungsversuche mit der Erprobung neuer Anbaumethoden und der Urbarmachung bis dahin ungenutzten Landes einher. Im Amt Oranienburg, das der aus den Niederlanden stammenden Kurfürstin Louise Henriette gehörte, bauten niederländische Kolonisten bereits seit 1651 eine Musterwirtschaft auf. Mit einigen friesischen bzw. nordniederländischen Siedlern wurden zur gleichen Zeit Verträge zur Anlage von Dörfern sowie zur Trockenlegung von Land in der Altmark und andernorts abgeschlossen. Die größtenteils reformierten Siedler erhielten zudem konfessionelle Zugeständnisse, etwa das Recht der reformierten Religionsausübung und der Pfarrerwahl, was freilich auch Konflikte mit der größtenteils lutherischen Landbevölkerung provozierte. ${ }^{52}$

Matthias Asche hat in seiner umfassenden Untersuchung zeigen können, dass auf diese Weise bis zum Beginn der 1680er Jahre die meisten Bauernstellen in der Prignitz wiederbesetzt werden konnten, dass aber durch erneute Kriegsereignisse in der Uckermark und im Land Ruppin die auch dort vorhandenen Ansätze mehrfach wieder zunichte gemacht wurden, sodass hier erst mit der Hugenotten- und Schweizerkolonisation ab 1685 die Mehrzahl der ländlichen Stellen wieder bewirtschaftet werden konnte..$^{53}$ In den folgenden Jahrzehnten setzte eine umfangreiche ländliche Siedlungspolitik ein, wobei die Übergänge von einer Repeuplierungspolitik nach

51 Niggemann, Peuplierung 2016, S. 171-176.

52 Asche, Neusiedler 2006, S. 261-285, 351-360, 431-434.

53 Ebd., S. 173-175, 195-205, 290-302. 
den Kriegsverlusten und einem forcierten und systematischen Landesausbau flieBend sind. Dahinter stand der Wunsch sowohl nach Bevölkerungsvermehrung als auch nach der Nutzung brachliegender Ressourcen zwecks Erhöhung der Staatseinnahmen. So bemühte sich die Regierung, in der Milchwirtschaft und Viehzucht erfahrene Schweizer Bauern für die Mark Brandenburg anzuwerben. 1685 konnten 14 Familien aus dem Berner Land in neu errichteten Dörfern bei Potsdam angesiedelt werden, und seit 1690 ließen sich Kolonisten aus den Kantonen Bern und Zürich schwerpunktmäßig im Land Ruppin und bei Potsdam nieder. Es handelte sich überwiegend um sehr kinderreiche Familien, die aufgrund ihrer Armut und der Landknappheit im Alpenraum aus der Schweiz auswanderten oder gar von den dortigen Almosenkammern zur Auswanderung gedrängt wurden. ${ }^{54}$

Von einer planmäßigen und auch quantitativ großangelegten Einwanderungspolitik kann dann aber erst im Kontext der Anwerbung von ,Glaubensflüchtlingen* die Rede sein. 1686 begann in der Folge des Potsdamer Edikts die Wiederbesiedlung zahlreicher wüstgefallener Dörfer in der Uckermark und im Land Ruppin mit Hugenotten. Nach dem verschärften konfessionspolitischen Vorgehen der französischen Regierung seit 1681 und insbesondere nach der Aufhebung des Edikts von Nantes im Oktober 1685 wanderten sie in verschiedene europäische Länder aus. 16.000 bis 20.000 von ihnen erreichten in der Regierungszeit der Kurfürsten Friedrich Wilhelm und Friedrich III./I. (1688-1713) Brandenburg-Preußen. ${ }^{55}$ Hinzu kamen noch wallonisch-stämmige Pfälzer, die im Zuge der französischen Besetzung der linksrheinischen Pfalz ab 1689 nach Brandenburg kamen. ${ }^{56}$

Die Ausweisung der Protestanten aus dem Fürstbistum Salzburg hingegen tangierte die Kurmark Brandenburg nur in sehr geringem Maße. Das am 2. Februar 1732 erlassene preußische Einladungspatent bezog sich vor allem auf das Herzogtum Preußen. Etwa 12.000 bis 15.000 Salzburger gelangten wahrscheinlich dorthin, nur einige wenige zogen in andere Gebiete der Monarchie. ${ }^{57}$ Noch unter König Friedrich Wilhelm I. (1713-1740) begann auch die Ansiedlung böhmischer Emigranten in Brandenburg und besonders in Berlin. Unter Friedrich II. wuchs die böhmische Kolonie in Berlin weiter an, und es wurden zusätzliche Ansiedlungen in Rixdorf, Nowawes bei Potsdam und in einigen anderen Orten geschaffen. ${ }^{58}$

$\mathrm{Zu}$ Peuplierungszwecken angeworben wurden auch kleinere Minderheitengruppen wie die Mennoniten, die schon erwähnte Herrnhuter Brüdergemeine oder sogenannte ,Inspirierte', die - oft nur temporär geduldet - von Ausweisungen bedroht und auf der Suche nach neuen Heimstätten waren. Insbesondere nach dem Dreißig-

54 Ebd., S. 218-221, 434f., 462-484.

55 Niggemann, Immigrationspolitik 2008, S. 54; Asche, Neusiedler 2006, S. 302-347.

56 Asche, Neusiedler 2006, S. $503 f$.

57 Walker, Handel 1997, S. 71-102.

58 Neugebauer, Zentralprovinz 2001, S. 129f.; Graffigna, Böhmen 1990, S. 506, 532-536; Jung, Weberkolonie 1997, S. 11-62. 
jährigen Krieg kamen zusammen mit reformierten Niederländern auch einige aus Polen einwandernde Mennoniten..$^{59}$

Ländliche Kolonisationsbemühungen prägten die Regierungszeit Friedrichs II. (1740-1786). In der Kurmark wurden schwerpunktmäßig deutsche Siedler aus preußischen oder benachbarten Territorien, jedoch auch aus Süddeutschland zur Urbarmachung eingesetzt. Im stark kriegszerstörten Schlesien und anderen östlichen Landesteilen waren es vor allem polnische Kolonisten, daneben aber auch Österreicher, Böhmen und sogar Griechen, die gezielt angesiedelt wurden. Insgesamt gründete die preußische Regierung mithilfe systematischer Werbungen wohl etwa 900 Kolonistendörfer. Dabei wurden nicht nur bäuerliche und kleinbäuerliche Hofstellen angelegt; zusätzlich entstanden auch ländliche Gewerbeansiedlungen, so etwa die Spinner- und Weberdörfer, in denen das Material für die Textilmanufakturen vorbereitet werden sollte. ${ }^{60}$

Im 18. Jahrhundert stand zudem die Neulandgewinnung im Zentrum der Bemühungen. Seit 1718 nahm Brandenburg-Preußen unter König Friedrich Wilhelm I. umfangreiche Meliorationsarbeiten in den Bruchgebieten an Havel und Rhin in Angriff. Überdies wurden die bereits zu Anfang des 17. Jahrhunderts in ersten Ansätzen begonnenen Arbeiten am Netze- und Dragebruch unter Friedrich Wilhelm I. wieder aufgegriffen. Mit Beginn der Meliorationen wurden Siedlungswillige durch öffentliche Ausschreibungen angeworben. Etwa tausend Arbeiter, darunter zahlreiche Soldaten, konnten zu diesem Zweck gewonnen und nach erfolgter Neulandgewinnung mit Kolonistenstellen versorgt werden. Während der Regierungszeit Friedrichs II. wurde insbesondere im ländlichen Bereich systematisch kolonisiert. Schon 1746 richtete sich das Interesse vor allem auf die noch unkultivierten, allerdings extensiv durchaus bewirtschafteten Bruchlandschaften an der Oder. Mit der Trockenlegung dieser Flusslandschaften wurde in großem Umfang neues Siedelland gewonnen, das an Pächter gegen die Verpflichtung vergeben wurde, Kolonisten anzusetzen. 1.200 Familien konnten auf diesem Wege in 43 neuen Dörfern angesiedelt werden, was allerdings von der in den Feuchtgebieten ansässigen Bevölkerung nicht ohne Widerstand hingenommen wurde. Nach dem Siebenjährigen Krieg wurden die Meliorations- und Drainagearbeiten in den Bruchgebieten an Rhin und Dosse sowie an Netze und Drage fortgesetzt. Mit dem Erwerb Westpreußens und des Netzedistrikts (1772) kamen die Trockenlegungen und Kolonisationen in den Warthebrüchen hinzu. Allein für die Kurmark Brandenburg warb die Regierung Friedrichs II. 100.000 neue Siedler an, die vor allem aus dem Südwesten Deutschlands, aber auch aus der Schweiz, aus Österreich, Böhmen, Polen und anderen Gegenden stammten. ${ }^{61}$

59 Niggemann, Peuplierung 2016, S. 191; Asche, Neusiedler 2006, S. 433.

60 Niggemann, Peuplierung 2016, 200f.

61 Asche, Neusiedler 2006, S. 377-402; Liesenberg, Kolonisation 2003, S. 35-102. 
Üblicherweise erhielten die hier genannten Einwanderergruppen Privilegien, die ihren kirchlichen, rechtlichen und wirtschaftlichen Status festlegten und zugleich als Anreiz für eine Ansiedlung konzipiert waren. Dementsprechend mussten sie so gefasst werden, dass sie auf potenzielle Einwanderer attraktiv wirkten. Ein typisches Element solcher Privilegien war die Steuerfreiheit für eine festgelegte Anzahl von Jahren. Darüber hinaus wurden die kostenlose Zuteilung von Bauland und Baumaterialien sowie freie Unterbringung für eine Übergangszeit zugesagt. Im kirchlichen Bereich erhielten viele der Siedler das Recht auf freie Religionsausübung sowie auf eigene Geistliche. Eine Besonderheit der brandenburgischen Hugenotten- und Pfälzeraufnahme war die Zusage einer eigenen Gerichtsbarkeit. Hier wie auch in einigen anderen Territorien entstand im Laufe der Zeit eine staatliche Sonderverwaltung für die französischen Kolonien. Unter Friedrich II. wurden den Siedlern ebenfalls weitreichende Privilegien zugestanden, so etwa die ,Enrollierungsfreiheit', das heißt die Befreiung vom Kriegsdienst bis in die dritte Generation der Siedler. Hinzu kamen Bekenntnisfreiheit und die Freiheit von der Leibeigenschaft. ${ }^{62}$

Trotz der massiven obrigkeitlichen Förderung waren Konflikte und Schwierigkeiten gerade in den Anfangsphasen dieser Kolonisationsprozesse an der Tagesordnung. Die einheimische Bevölkerung, die in den wenig erschlossenen - aus Sicht der Regierung nicht effizient bewirtschafteten - Regionen durchaus extensive Wirtschaft betrieben, sahen ihre hergebrachte Lebensweise durch Eindeichungen, Trockenlegungen und Anlage von Kolonistendörfern gefährdet. Im Zuge von Protesten zerstörten sie Deiche, rissen Zäune ein und vernichteten Feldfrüchte. Selbst da, wo Kolonisten und Einheimische, wie etwa bei der Hugenottenansiedlung im ländlichen Brandenburg in gemeinsamen Dörfern lebten, blieben die Gemeinden teilweise lange unter sich. So lassen sich bei den Hugenotten noch bis in die dritte Generation hinein relativ endogame Heiratsmuster feststellen, ebenso wie Versuche, zumindest formal an der französischen Sprache als Gottesdienst- und Verwaltungssprache festzuhalten. Es war also nicht nur Exklusion durch die einheimische Bevölkerung, sondern auch das Festhalten an einer eigenen Gruppenidentität wie auch an den obrigkeitlich zugesagten Privilegien, die ein gewisses Maß an Abschließung hervorbrachten. Und schließlich blieb auch die französisch-reformierte Konfession ein trennendes Element, das freilich weniger konfliktträchtig war, als man lange annahm. ${ }^{63}$

Hinzuweisen ist natürlich auch auf die Tatsache, dass viele der ländlichen Kolonien von den Obrigkeiten zunehmend kritisch gesehen wurden. Derselbe König Friedrich Wilhelm I., der sich so intensiv um die Repeuplierung der landlichen Ge-

62 Asche, Neusiedler 2006, S. 510-513; Niggemann, Immigrationspolitik 2008, S. 101-106, 369-372; Ders., Peuplierung 2016, S. 174f., 192-196, 200 f.

63 Niggemann, Immigrationspolitik 2008, S. 448-453. 
genden Ostpreußens bemühte, hatte für ländliche Hugenottensiedlungen nichts übrig. Und auch den Schweizer Kolonisten wurde vorgehalten, sie seien zu faul, um sich aus eigener Kraft zu ernähren. ${ }^{64}$ Geradezu paradox mutet zudem die unter Friedrich Wilhelm I. durchgeführte Ausweisung der Mennoniten an, die zeitgleich mit der Salzburger Ansiedlung stattfand. Begründet wurde diese Maßnahme vor allem mit der Verweigerung des Militärdienstes durch diese dem strikten Pazifismus verpflichtete Gruppe. ${ }^{65}$ Solche Vorgänge waren insgesamt keine Seltenheit und verweisen auf den Utilitarismus der Einwanderungspolitik, in deren Logik es lag, Bevölkerungsgruppen in nützliche und weniger nützliche zu kategorisieren.

\subsection{Städtische Neusiedler, Neustädte und Residenzausbau}

Besondere Aufmerksamkeit richteten die Merkantilisten stets auf das städtische Umfeld, insbesondere auf Handel und Gewerbe. ,Peuplierung، und Gewerbeförderung waren in der Regel eng miteinander verflochten. Die Ansiedlung von Menschen diente der Bevölkerungsvermehrung, war aber gleichzeitig auch eine Maßnahme zur Förderung der Wirtschaft, insbesondere zur Weiterentwicklung neuer Betriebsformen und zur Herstellung bislang nur im Ausland produzierter Güter. Besonders interessiert waren die von merkantilistischen Theorien beeinflussten Landesherren an der Niederlassung von Manufakturen. Abgesehen von der Möglichkeit, hier sowohl Massenwaren - etwa für den Militärbedarf - als auch Luxusgüter für den Hof produzieren zu lassen, konnten in den Manufakturen ebenso zahlreiche Arbeiter beschäftigt werden. ${ }^{66}$ Dabei spielte die großangelegte Aufnahme religiös Verfolgter eine wichtige Rolle.

In diesem Zusammenhang bildete das 1671 durch Kurfürst Friedrich Wilhelm erfolgte Aufnahmeangebot für 50 aus Wien und Niederösterreich vertriebene jüdische Familien gewissermaßen den Auftakt für großangelegte planmäßige Wiederaufsiedlungsversuche in den kriegszerstörten Städten. Allerdings konnten die Juden nur bedingt von den Einwandererprivilegien, die für christliche Gruppen galten, profitieren, enthielt das Aufnahmeangebot doch erhebliche Restriktionen. Es wurden ihnen zwar eine gewisse Eigenständigkeit in der Gerichtsbarkeit sowie das Recht zur privaten Religionsausübung zugesagt, doch durften sie keine Synagogen errichten. Überhaupt blieb ihre Rechtsstellung bis zum preußischen Emanzipationsedikt von 1812 prekär. Noch unter Friedrich II. wurden im Generalreglement von 1750 zahlreiche rechtliche Einschränkungen und Diskriminierungen verankert. ${ }^{67}$

64 Asche, Neusiedler 2006, S. 241 mit Anm. 882 sowie S. 340f., 529-552.

65 Walker, Handel 1997, S. 85 .

66 Niggemann, Immigrationspolitik 2008, S. 289 f.

67 Jersch-Wenzel, Juden 1978, S. 26-39; Scheiger, Juden 1990, S. 158, 164-171, 181. 
Große Manufakturen, insbesondere im Bereich der Textilherstellung, entstanden im Zuge der Hugenotteneinwanderung in Berlin, Magdeburg und Halle. Auch unter den Schweizer Einwanderern befanden sich Handwerker und Manufakturisten, die in Berlin-Cölln, Potsdam, Neustadt-Eberswalde und Prenzlau angesiedelt wurden. ${ }^{68}$ Bei der Anlage dieser Großbetriebe spielten landesherrliche Zuschüsse und Kredite eine zentrale Rolle. Über die finanziellen Zuwendungen hinaus erhielten die Manufakturisten in der Regel auch Gebäude oder zumindest Baumaterial. Vielfach wurden ihnen sogar Produktionsanlagen, wie Lohmühlen, aus landesherrlichen Mitteln erbaut. ${ }^{69}$ Hinzu kamen umfangreiche, oft über die allgemeinen Einwandererprivilegien hinausgehende Steuer- und Zollvergünstigungen. Dennoch erwiesen sich viele dieser Betriebe als unwirtschaftlich, was auch an den unterentwickelten Absatzstrukturen lag. Streitigkeiten und langwierige Prozesse der Manufakturisten untereinander waren - besonders auffällig im Falle der Hugenotten - die Folge. $^{70}$

In der Praxis blieben größere Manufakturbetriebe in Brandenburg die Ausnahme. Auch unter den Hugenotten, die in die Territorien der Kurfürsten kamen, befanden sich nur wenige, die solche Betriebe eröffneten. Die Mehrzahl der in den Städten angesiedelten Hugenotten betätigte sich im Rahmen des traditionellen Kleinhandwerks, wo sie sich spätestens nach Ablauf der in den Einwandererprivilegien festgelegten Freijahren in aller Regel den ortsüblichen zünftigen Strukturen anpassen mussten. Diese Anpassung verlief nicht immer konfliktfrei, zumal sich die eingesessenen Zünfte auf ihre korporativen Privilegien berufen konnten und den Anspruch erhoben, den Zugang zum Handwerk ohne obrigkeitliche Einmischung selbst zu regulieren. ${ }^{71}$

Auch städtische Einwanderergruppen behielten zumeist noch lange eine spezifische Gruppenidentität bei, besonders wenn sie, wie etwa die Hugenotten oder die Pfälzer, Privilegiengemeinschaften bildeten oder gar ein ausgesprochen elitäres Bewusstsein entwickelten, wie das etwa bei den Berliner Hugenotten der Fall war, die über eigene Schulen sowie intellektuelle Zirkel verfügten und eine besondere Nähe zum Hof pflegten. Die Kirchengemeinden bildeten Zentren der eigenen kulturellen Identität, und entsprechend lange dauerte es, bis exogam geheiratet wurde und der Übergang in die einheimischen Kirchengemeinden vollzogen wurde. Freilich ist bei diesen Vorgängen zu bedenken, dass Integration in einem modernen Sinne zu keinem Zeitpunkt im 16., 17. und 18. Jahrhundert als politisches oder gesellschaftliches Ziel auch nur denkbar war. Vielmehr dachte die vormoderne Gesellschaft in korpo-

68 Asche, Neusiedler 2006, S. 245f.

69 Beispiele bei: Jersch-Wenzel, Juden 1978, S. 79f.; Niggemann, Immigrationspolitik 2008, S. 290-294.

70 Niggemann, Immigrationspolitik 2008, S. 304-308, 315-318.

71 Ebd., S. 320-354. 
rativen Strukturen. Die französische oder Pfälzer Kolonie waren Rechtsgebilde, die neben anderen rechtlich definierten und korporativ strukturierten gesellschaftlichen Formationen standen, während die Idee einer homogenen Untertanengesellschaft erst im 19. Jahrhundert wirklich vorherrschend wurde. ${ }^{72}$

Der Regelfall der städtischen Kolonisation war die Zusiedlung der Einwanderer in den bestehenden Städten. Insbesondere in den stark kriegsverwüsteten Städten wurden den Einwanderern wüste Hausstellen oder instand zu setzende Häuser eingeräumt. Zum Teil erfolgte die Ansiedlung allerdings auch innerhalb planmäßig ausgebauter Vorstädte, insbesondere bei den Residenzen. Ab 1660 begann der Ausbau Potsdams als Zweitresidenz des Kurfürsten Friedrich Wilhelm von Brandenburg. Unter König Friedrich II. folgte dann die weitere Ausgestaltung durch den Bau des Schlosses Sanssouci und des Neuen Palais. Zuvor schon war Potsdam unter Friedrich Wilhelm I. zu einem wichtigen Garnisonsstandort geworden. Auch mit dem Ausbau Berlins wurde in der Zeit Kurfürst Friedrich Wilhelms begonnen, zuerst mit der Befestigung und Privilegierung des Friedrichswerders (1662), dann mit dem Bau der Dorotheenstadt (seit 1673) und der Friedrichsstadt (seit 1688). Hier wurden einerseits ,Glaubensflüchtlinge' wie die Hugenotten angesiedelt, es wurden andererseits aber auch durch Privilegien neue Einwohner aus dem Reich angeworben. ${ }^{73}$

Berlin wurde Ende des 18. Jahrhunderts neben Koblenz darüber hinaus zu einem Zentrum französischer Revolutionsflüchtlinge (,Émigrés'). Dabei handelte es sich vielfach um Adelige, die zum Teil auch als Offiziere in preußischen Diensten gegen das revolutionäre Frankreich kämpften. Mit dem Ende der Revolutionsepoche kehrte jedoch ein großer Teil dieser Flüchtlinge wieder nach Frankreich zurück. ${ }^{74}$ Dennoch wird man diese zweite Welle französischsprachiger Immigranten nach den Hugenotten für die kulturelle Entwicklung in der Mark Brandenburg nicht unterschätzen dürfen. Von der landesgeschichtlichen Forschung sind die Émigrés der Revolutionsära im Gegensatz zu den Hugenotten vielleicht noch zu wenig beachtet worden.

\subsection{Urbanisierung und Industrialisierung}

Das Wachstum der Städte setzte sich im 19. Jahrhundert verstärkt fort. Einerseits hatte dies zu tun mit der industriellen Entwicklung und den damit einhergehenden Hoffnungen gerade der ländlichen Unterschichten auf ein Auskommen in der städtischen Industrie, andererseits aber auch mit den Entwicklungen hin zu einer Freizügigkeit innerhalb des preußischen Staatswesens. Schon seit Beginn des 18. Jahrhun-

72 Ebd., S. $393 f$.

73 Niggemann, Peuplierung 2016, S. 213.

74 Höpel, Emigranten 2000. 
derts wurden die Abzugsgelder bei einem Ortswechsel innerhalb der brandenburgisch-preußischen Territorien allmählich abgeschafft, und das „Allgemeine Landrecht für die Preußischen Staaten“ von 1794 beseitigte zudem die Steuer auf mitgeführtes Vermögen. Auch die sogenannte ,Bauernbefreiung sorgte seit dem frühen 19. Jahrhundert dafür, dass diejenigen Teile der ländlichen Bevölkerung, die bislang erbuntertänig und schollengebunden waren, jetzt über die rechtlichen Voraussetzungen für mehr Mobilität verfügten. Gleichzeitig bemühte man sich auch um die Begrenzung der Auswanderung aus Brandenburg, insbesondere nach Übersee. ${ }^{75}$

Im Zuge der Industrialisierung entwickelte sich vor allem Berlin zu einem Zentrum des Maschinenbaus und der Metallindustrie und zog damit verstärkt Zuwanderer an, die die Stadt im Laufe des 19. Jahrhunderts schnell zu einer Großstadt werden ließ. Der Großteil kam aus der Mark Brandenburg, sodass die brandenburgische Binnenmigration eine beträchtliche Rolle spielte. Zwar ist auch von Rückwanderungen in die ländlichen Regionen auszugehen, doch insgesamt scheint schon im Laufe des 19. Jahrhunderts die ländliche Mark von einer starken Abwanderung in die Großstadt geprägt gewesen zu sein. Zu einer beträchtlichen Zuwanderung kam es zudem aus dem östlichen Europa. Polen, aber auch Tschechen, Slowaken und Russen kamen nach Berlin, wo sie als Handwerker und Industriearbeiter tätig waren; polnische Frauen kamen oft als Dienstbotinnen in den bürgerlichen Haushalten unter oder betätigten sich als Näherinnen, im Reinigungsgewerbe und in der Lebensmittelherstellung. Diese Zuwanderergruppen prägten vor allem die schnell wachsenden Wohnviertel der Mittel- und Unterschichten. ${ }^{76}$ Daneben spielten im ländlichen Raum - in Brandenburg ebenso wie in den übrigen agrarischen Provinzen Preußens - die polnischen landwirtschaftlichen Saisonarbeiter eine wichtige Rolle.

\subsection{Der Zweite Weltkrieg und die Folgen}

Mit der Etablierung der nationalsozialistischen Diktatur begann ein neuer Abschnitt in der Migrationsgeschichte Brandenburgs wie auch anderer Regionen des Deutschen Reiches. Politische Gegner, Juden, Sinti und Roma sowie andere unerwünschte Gruppen wurden zunehmend in ihren Handlungsspielräumen eingeschränkt, terrorisiert, verhaftet, deportiert und schließlich ermordet. Andere konnten sich durch rechtzeitige Emigration der Terrorherrschaft entziehen. Auch die vom ,Dritten Reich“ praktizierte Zwangsarbeit gehört in diesen Zusammenhang. In Oranienburg wurde zunächst auf einem alten Brauereigelände eines der frühesten nationalsozialisti-

75 Hitzer, Freizügigkeit 2016, S. 247f., 251-257; Hartmann, Polen 1990, S. 625; Plaß, Massenmigration 2016.

76 Hartmann, Polen 1990. 
schen Konzentrationslager errichtet. Hier wurden bis Juli 1934 von der SA vor allem politische Häftlinge aus dem Reich interniert. Im Zuge des sogenannten Röhm-Putsches und der Auflösung der SA wurde das Lager geschlossen. 1936 entstand dann bei Oranienburg das Konzentrationslager Sachsenhausen, wohin in den Folgejahren etwa 200.000 Menschen aus unterschiedlichen Regionen deportiert wurden. Mehrere zehntausend Häftlinge wurden hier ermordet. ${ }^{77}$ Flucht, aber auch Deportation lassen sich als Formen der (Zwangs-)Migration beschreiben und sind somit Teil des Migrationsgeschehens in der Region, auch wenn sie zweifellos einen Sonderfall darstellen.

Gegen Ende des Krieges setzte auch die Flucht und Vertreibung aus den ehemaligen Ostgebieten des Reiches ein, wobei Brandenburg sowohl Ziel- als auch Ausgangsgebiet dieser Migrationsströme war. Aus Preußen, Pommern und Schlesien kamen Hunderttausende auch nach Brandenburg, und aus den östlichen Gebieten Brandenburgs (der Neumark) flohen Menschen über die Oder aus den nunmehr unter polnischer Verwaltung stehenden Regionen. Die Vertriebenen wurden zunächst in Lagern untergebracht, bevor sie schließlich weiterverteilt wurden. Allein in Brandenburg existierten vier Auffanglager sowie 16 Kreis- und Quarantänelager. ${ }^{78}$

Nach der Gründung der DDR hielt die Abwanderung von Menschen in die neugegründete Bundesrepublik Deutschland weiter an. Zwar ließ der Migrationsstrom mit der Konsolidierung des neuen Staates, aber vor allem auch aufgrund der zunehmenden Unpassierbarkeit der Westgrenze der DDR nach, doch mit den wachsenden wirtschaftlichen Problemen in den 1980er Jahren verstärkte er sich wieder, wobei jetzt auch die Zahl der regulären Ausreisegenehmigungen anstieg. Darüber hinaus kam es in Westberlin seit den 1960er Jahren zu einem starken Zuzug vor allem türkischer Arbeitsmigranten, von denen sich viele dauerhaft niederließen und in den 1970er Jahren ihre Familien nachholten. So entstanden zahlenmäßig starke türkische Gemeinden und ein migrantisch geprägtes kulturelles Milieu, das einen nicht $\mathrm{zu}$ unterschätzenden Einfluss - etwa in Form von mitgebrachten Produkten und kulinarischen Gewohnheiten - auch auf die eingesessene Bevölkerung ausübte. In Ostberlin und Brandenburg kam es zeitgleich zu einer Zuwanderung aus sozialistischen Staaten. Insbesondere aus Vietnam und Mosambique, aber auch aus dem benachbarten Polen warb die DDR Arbeitskräfte an, die allerdings weitgehend separiert von der einheimischen Bevölkerung angesiedelt wurden. ${ }^{79}$ Auch die Präsenz sowjetischer Truppen gehört in den Kontext des Migrationsgeschehens - die russische Kolonie Alexandrowka in Potsdam zeugt etwa davon.

77 Bade/Oltmer, Normalfall 2004, S. 41-46; Rass, Wanderungslenkung 2016; Schmiechen-Ackermann, Rassismus 2016; Benz/Distel, Ort 2006; Morsch, Konzentrationslager 1994.

78 Franzen, Migration 2016, S. 722-732; Oehlsen, Vertriebenenlager 2006.

79 Bade/Oltmer, Normalfall 1994, S. 90-95. 
$\mathrm{Zu}$ beobachten ist im Hinblick auf die Binnenwanderung eine anhaltende $\mathrm{Ab}$ wanderung aus den ländlichen Regionen, die auch über die Wende von 1989 und die Wiedervereinigung von 1990 hinausgeht. Während insbesondere Berlin einen starken Zuzug zu verzeichnen hat, verlassen viele Menschen das ländliche Brandenburg, um in anderen Regionen der Bundesrepublik ihr Auskommen zu finden. Dabei bleibt bis heute die regionale Prägung von Migrationsprozessen deutlich. Die strukturellen Voraussetzungen und Lebensbedingungen vor Ort haben wesentlichen Einfluss auf $\mathrm{Zu}$ - und Abwanderungen sowie auf die Ausformungen des Migrationsgeschehens. Die politischen Rahmenbedingungen, die Prioritätensetzungen der Obrigkeiten und die sich herausbildenden Migrationsregime waren und sind somit vor allem auch im Kontext der regionalen Verhältnisse zu verstehen.

\section{Literatur}

Asche, Matthias: Neusiedler im verheerten Land. Kriegsfolgenbewältigung, Migrationssteuerung und Konfessionspolitik im Zeichen des Landeswiederaufbaus. Die Mark Brandenburg nach den Kriegen des 17. Jahrhunderts. Münster 2006.

Asche, Matthias/Niggemann, Ulrich (Hrsg.): Das leere Land - Historische Narrative von Einwanderergesellschaften. Stuttgart 2015 (Historische Mitteilungen. Beihefte 92).

Assing, Helmut: Die Landesherrschaft der Askanier, Wittelsbacher und Luxemburger (Mitte des 12. bis Anfang des 15. Jahrhunderts. In: Materna, Ingo/Ribbe, Wolfgang (Hrsg.): Brandenburgische Geschichte. Berlin 1995, S. 85-168.

Bade, Klaus J. u.a. (Hrsg.): Enzyklopädie Migration in Europa vom 17. Jahrhundert bis zur Gegenwart. Paderborn 2007.

Bade, Klaus J./Oltmer, Jochen: Deutschland. In: Bade, Klaus J. u.a. (Hrsg.): Enzyklopädie Migration in Europa vom 17. Jahrhundert bis zur Gegenwart. Paderborn 2007, S. 141-170.

Bade, Klaus J.: Sozialhistorische Migrationsforschung. In: Ders.: Sozialhistorische Migrationsforschung. Göttingen 2004 (Studien zur historischen Migrationsforschung, 13), S. 27-48.

Bade, Klaus J./Oltmer, Jochen: Normalfall Migration. Bonn 2004 (Zeitbilder, 15).

Benz, Wolfgang/Distel, Barbara (Hrsg.): Der Ort des Terrors. Geschichte der nationalsozialistischen Konzentrationslager. Bd. 3: Sachsenhausen, Buchenwald. München 2006.

Böcker, Heidelore: Die Festigung der Landesherrschaft durch die hohenzollernschen Kurfürsten und der Ausbau der Mark zum fürstlichen Territorialstaat während des 15. Jahrhunderts. In: Materna, Ingo/Ribbe, Wolfgang (Hrsg.): Brandenburgische Geschichte. Berlin 1995, S. $169-230$.

Bösl, Elsbeth/Kramer, Nicole/Linsinger, Stephanie: Die vielen Gesichter der Zwangsarbeit: „Ausländereinsatz“ im Landkreis München 1939-1945. München 2005.

Brinkmann, Tobias: Von der Gemeinde zur "Community“, Jüdische Einwanderer in Chicago 18401900. Osnabrück 2002.

Deutinger, Stephan: Vom Agrarland zum High-Tech-Staat: zur Geschichte des Forschungsstandorts Bayern 1945-1980. München/Wien 2001.

Düwell, Frank: Europäische und internationale Migration. Einführung in historische, soziologische und politische Analysen. Münster 2006.

Fabbro, René del: Transalpini. Italienische Arbeitswanderung nach Süddeutschland im Kaiserreich 1870-1918. Osnabrück 1996. 
Faltin, Sigrid: Die Auswanderung aus der Pfalz nach Nordamerika im 19. Jahrhundert. Unter besonderer Berücksichtigung des Landkommissariates Bergzabern. Frankfurt a.M. u.a. 1987.

Fassl, Peter (Hrsg.): Geschichte und Kultur der Juden in Schwaben. 3 Bde. Sigmaringen/Stuttgart 1994-2007.

Fegert, Friedemann: „Ihr ghönt es Eich gar nicht vorstelen wie es in Amerigha zu ged.": Auswanderung aus den jungen Rodungsdörfern des Passauer Abteilandes nach Nordamerika seit der Mitte des 19. Jahrhunderts. Freyung ${ }^{2} 2014$.

Franzen, K. Erik: Migration als Kriegsfolge. Instrumente und Intentionen staatlicher Akteure nach 1945. In: Oltmer, Jochen (Hrsg.): Handbuch Staat und Migration in Deutschland seit dem 17. Jahrhundert. Berlin u.a. 2016, S. 721-739.

Frevert, Ute/Oltmer, Jochen (Hrsg.): Europäische Migrationsregime. Göttingen 2009 (Geschichte und Gesellschaft, 35/1).

Friederich, Christoph: 300 Jahre Hugenottenstadt Erlangen: Vom Nutzen der Toleranz. Ausstellung im Stadtmuseum Erlangen. Nürnberg 1986.

Gestrich, Andreas/Krauss, Marita (Hrsg.): Migration und Grenze. Stuttgart 1998.

Gestrich, Andreas/Krauss, Marita (Hrsg.): Zurückbleiben. Der vernachlässigte Teil der Migrationsgeschichte. Stuttgart 2006.

Gotthard, Axel: Der Augsburger Religionsfrieden. Münster 2004.

Graffigna, Eva-Maria: Böhmen in Berlin. In: Jersch-Wenzel, Stefi/John, Barbara (Hrsg.): Von Zuwanderern zu Einheimischen. Hugenotten, Juden, Böhmen, Polen in Berlin. Berlin 1990, S. 491-591.

Grossmann, Anton J.: Fremd- und Zwangsarbeiter in Bayern 1939-1945. In: Vierteljahreshefte für Zeitgeschichte 34 (1986), Heft 4, S. 481-521.

Hahn, Peter-Michael: Geschichte Brandenburgs. München 2009.

Hamm, Margot/Henker, Michael/Brockhoff, Evamaria (Hrsg.): Good Bye Bayern. Grüß Gott Amerika. Auswanderung aus Bayern nach Amerika seit 1683. Augsburg 2004.

Härter, Karl: Grenzen, Streifen, Pässe und Gesetze. Die Steuerung von Migration im frühneuzeitlichen Territorialstaat des Alten Reiches (1648-1806). In: Oltmer, Jochen (Hrsg.): Handbuch Staat und Migration in Deutschland seit dem 17. Jahrhundert. Berlin u.a. 2016, S. 45-86.

Hartmann, Gottfried: Polen in Berlin. In: Jersch-Wenzel, Stefi/John, Barbara (Hrsg.): Von Zuwanderern zu Einheimischen. Hugenotten, Juden, Böhmen, Polen in Berlin. Berlin 1990, S. 593-800.

Hartmann, Peter Claus/Schmid, Alois (Hrsg.): Bayern in Lateinamerika. Transatlantische Verbindungen und interkultureller Austausch. München 2011.

Heese, Henning: Einleitende Bemerkungen zur Entstehung und Entwicklung der Schweizer Kolonie im Golmischen Bruch bei Potsdam. In: Ders. (Hrsg.): 300 Jahre Schweizer Kolonie am Golmischen Bruch bei Potsdam 1685-1985. St. Augustin-Hangelar 1985, S. 5-43.

Hehemann, Rainer: "... Jederzeit gottlose böse Leute“ - Sinti und Roma zwischen Duldung und Vernichtung. In: Bade, Klaus J. (Hrsg.): Deutsche im Ausland - Fremde in Deutschland. Migration in Geschichte und Gegenwart. München 1992, S. 271-277.

Heinrich, Gerd: Geschichte Preußens. Staat und Dynastie. Frankfurt a.M. U.a. 1981.

Helbich, Wolfgang u.a.: Briefe aus Amerika. Deutsche Auswanderer schreiben aus der Neuen Welt 1830-1930. München 1988.

Hitzer, Bettina: Freizügigkeit als Reformergebnis und die Entwicklung von Arbeitsmärkten. In: Oltmer, Jochen (Hrsg.): Handbuch Staat und Migration in Deutschland seit dem 17. Jahrhundert. Berlin u.a. 2016, S. 245-289.

Höpel, Thomas: Emigranten der Französischen Revolution in Preußen 1789-1806. Eine Studie in vergleichender Perspektive. Leipzig 2000 (Deutsch-französische Kulturbibliothek, 17). 
Jersch-Wenzel, Stefi: Juden und Franzosen in der Wirtschaft des Raumes Berlin/Brandenburg zur Zeit des Merkantilismus. Berlin 1978 (Einzelveröffentlichungen der Historischen Kommission zu Berlin, 23).

Jung, Karin C.: Die Böhmische Weberkolonie Nowawes 1751-1767 in Potsdam-Babelsberg. Bauliche und städtebauliche Entwicklung. Berlin 1997.

Kasberger, Erich: Lehm - Gold der Erde. In: Knauer-Nothaft, Christl/Kasberger, Erich (Hrsg.): Berg am Laim. München 2007, S. 211-230.

Kluxen, Andrea M./Krieger, Julia/May, Andrea i.A. des Bezirks Mittelfranken (Hrsg.): Fremde in Franken: Migration und Kulturtransfer. Würzburg 2016.

Knabe, Wolfgang: Aufbruch in die Ferne. Deutsche Auswanderungen zwischen 1803 und 1914 am Beispiel Bayerisch-Schwaben. Berlin (Ost) 1992.

Krauss, Marita: Arbeitswanderer aus Italien in Schwaben vom Ende des 19. Jahrhunderts bis nach dem Zweiten Weltkrieg. In: Zeitschrift des historischen Vereins für Schwaben. Augsburg 2010, S. 201-216.

Krauss, Marita (Hrsg.): Die bayerischen Kommerzienräte. Eine deutsche Wirtschaftselite 18801928. München 2016.

Krauss, Marita: Heimkehr in ein fremdes Land. Geschichte der Remigration nach 1945. München 2001.

Krauss, Marita (Hrsg.): Integrationen. Vertriebene in den deutschen Ländern nach 1945. Göttingen 2008.

Krauss, Marita: Nachkriegskultur in Bayern. Münchner städtische Kulturpolitik 1945-1954. München 1985, S. 196-200.

Krauss, Marita/Sonnabend, Holger (Hrsg.): Frauen und Migration. Stuttgart 2001.

Liesenberg, Carsten: Die Kolonisation des Oderbruchs. Planung, Ablauf und Ergebnisse eines absolutistischen Besiedlungskonzeptes aus baulicher Sicht. Diss. Weimar 2003.

Maidl, Peter: Auswanderung nach Übersee. Bd. 1: Studien zur bayerisch-schwäbischen Auswanderung nach Nordamerika im 19. Jahrhundert. Augsburg 1993.

Maidl, Peter: „hier ißt man anstadt Kardofln und Schwarzbrodt Pasteten...“. Die deutsche Überseewanderung des 19. Jahrhunderts in Zeitzeugnissen. Augsburg 1993.

Morsch, Günter (Hrsg.): Konzentrationslager Oranienburg. Berlin 1994 (Schriftenreihe der Stiftung Brandenburgische Gedenkstätten, 3).

Neugebauer, Wolfgang: Zentralprovinz des Absolutismus. Brandenburg im 17. und 18. Jahrhundert. Berlin 2001 (Bibliothek der brandenburgischen und preußischen Geschichte, 5).

Niggemann, Ulrich: Immigrationspolitik zwischen Konflikt und Konsens. Die Hugenottenansiedlung in Deutschland und England (1681-1697). Köln/Weimar/Wien 2008 (Norm und Struktur, 33).

Niggemann, Ulrich: ,Peuplierung' als merkantilistisches Instrument: Privilegierung von Immigranten und staatlich gelenkte Ansiedlungen. In: Oltmer, Jochen (Hrsg.): Handbuch Staat und Migration in Deutschland seit dem 17. Jahrhundert. Berlin u.a. 2016, S. 171-218.

Oehisen, Sven O.: Vertriebenenlager in Brandenburg 1945-1953. Potsdam 2006 (Brandenburgische Historische Hefte, 18).

Oltmer, Jochen: Einführung: Europäische Migrationsverhältnisse und Migrationsregime in der Neuzeit. In: Frevert, Ute/Oltmer, Jochen (Hrsg.): Europäische Migrationsregime. Göttingen 2009 (Geschichte und Gesellschaft, 35/1), S. 5-27.

Partenheimer, Lutz: Albrecht der Bär. Gründer der Mark Brandenburg und des Fürstentums Anhalt. Köln u.a. 2011.

Plaß, Uwe: Überseeische Massenmigration zwischen politischem Desinteresse und Staatsintervention. In: Oltmer, Jochen (Hrsg.): Handbuch Staat und Migration in Deutschland seit dem 17. Jahrhundert. Berlin u.a. 2016, S. 291-315.

Prinz, Friedrich: Geschichte Bayerns. München 1997. 
Prinz, Friedrich: Integration und Neubeginn. Dokumentation über die Leistungen des Freistaates Bayern und des Bundes zur Eingliederung der Wirtschaftsbetriebe der Vertriebenen und Flüchtlinge, hrsg. v. Bayerischen Staatsministerium für Arbeit und Sozialordnung. München 1984.

Prinz, Friedrich/Krauss, Marita (Hrsg.): München - Musenstadt mit Hinterhöfen. Die Prinzregentenzeit 1888-1912. München 1988.

Raithel, Thomas: „Kommt bald nach...“. Auswanderung aus Bayern nach Amerika 1683-2003. In: Hamm, Margot/Henker, Michael/Brockhoff, Evamaria (Hrsg.): Good Bye Bayern. Grüß Gott Amerika. Auswanderung aus Bayern nach Amerika seit 1683. Augsburg 2004, S. 23-36.

Rass, Christoph: Wanderungslenkung und Kriegsvorbereitung 1933-1939. In: Oltmer, Jochen (Hrsg.): Handbuch Staat und Migration in Deutschland seit dem 17. Jahrhundert. Berlin u.a. 2016, S. 537-571.

Rottmann, Eduard Friedrich (Hrsg.): Die Lehre von der Aus- und Einwanderung im Königreiche Bayern und das Verfahren und der Dienst der Verwaltungsämter diesseits des Rheins in Ausund Einwanderungssachen. Würzburg 1862.

Schaub, Hans: Auswanderung aus Oberfranken nach den Vereinigten Staaten von Amerika. Diss. Bamberg 1989.

Scheiger, Brigitte: Juden in Berlin. In: Jersch-Wenzel, Stefi/John, Barbara (Hrsg.): Von Zuwanderern zu Einheimischen. Hugenotten, Juden, Böhmen, Polen in Berlin. Berlin 1990, S. 153-488.

Schmiechen-Ackermann, Detlef: Rassismus, politische Verfolgung und Migration: Ausgrenzung und Austreibung ,unerwünschter" Gruppen aus dem nationalsozialistischen Deutschland. In: Oltmer, Jochen (Hrsg.), Handbuch Staat und Migration in Deutschland seit dem 17. Jahrhundert. Berlin u.a. 2016, S. 574-642.

Schnabel, Werner Wilhelm: Österreichische Exulanten in oberdeutschen Reichsstädten. Zur Migration von Führungsschichten im 17. Jahrhundert. München 1992.

Schnabel, Werner Wilhelm: Österreichische Glaubensflüchtlinge in Franken. Integration und Assimilation von Exulanten im 17. Jahrhundert. In: Blessing, Werner K./Weiss, Dieter J. (Hrsg.): Franken. Vorstellungen und Wirklichkeit in der Geschichte. Neustadt a.d. Aisch 2003. S. 161-178.

Statistisches Landesamt (Hrsg.): Bayern und seine Gemeinden unter dem Einfluß der Wanderungen während der letzten 50 Jahre. München 1912 (Beiträge zur Statistik des Königreichs Bayern, 69).

Treml, Manfred/Kirmeier, Josef (Hrsg.): Geschichte und Kultur der Juden in Bayern. Aufsätze. München 1988 (Veröffentlichungen zur Bayerischen Geschichte und Kultur, 17).

Uhlig, Otto: Die Schwabenkinder aus Tirol und Vorarlberg. Innsbruck ${ }^{4} 2003$.

Ullmann, Sabine: Nachbarschaft und Konkurrenz. Juden und Christen in den Dörfern der Markgrafschaft Burgau 1650 bis 1750. Göttingen 1999 (Veröffentlichungen des Max-Planck-Instituts für Geschichte, 151).

Vagts, Alfred: Deutsch-Amerikanische Rückwanderung. Probleme - Phänomene - Statistik Politik - Soziologie - Biographie. Heidelberg 1960.

Walker, Mack: Der Salzburger Handel. Vertreibung und Errettung der Salzburger Protestanten im 18. Jahrhundert. Göttingen 1997 (Veröffentlichungen des Max-Planck-Instituts für Geschichte, 131).

Wetzel, Juliane/Königseder, Angelika: Lebensmut im Wartesaal. Die jüdischen DPs (Displaced Persons) im Nachkriegsdeutschland. Frankfurt a.M. 1994. 